\title{
LARGE DISPERSION, AVERAGING AND ATTRACTORS: THREE 1D PARADIGMS
}

\author{
ANNA KOSTIANKO ${ }^{1}$, EDRISS TITI ${ }^{2,3}$ AND SERGEY ZELIK ${ }^{1}$
}

\begin{abstract}
The effect of rapid oscillations, related to large dispersion terms, on the dynamics of dissipative evolution equations is studied for the model examples of the 1D complex GinzburgLandau and the Kuramoto-Sivashinsky equations. Three different scenarios of this effect are demonstrated. According to the first scenario, the dissipation mechanism is not affected and the diameter of the global attractor remains uniformly bounded with respect to the very large dispersion coefficient. However, the limit equation, as the dispersion parameter tends to infinity, becomes a gradient system. Therefore, adding the large dispersion term actually suppresses the non-trivial dynamics. According to the second scenario, neither the dissipation mechanism, nor the dynamics are essentially affected by the large dispersion and the limit dynamics remains complicated (chaotic). Finally, it is demonstrated in the third scenario that the dissipation mechanism is completely destroyed by the large dispersion, and that the diameter of the global attractor grows together with the growth of the dispersion parameter.
\end{abstract}

\section{Contents}

1. Introduction 1

2. Preliminaries 3

2.1. Dispersive Ginzburg-Landau equation $\quad 4$

2.2. Classical Ginzburg-Landau equation $\quad 6$

2.3. Kuramoto-Sivashinsky equation 7

3. Global attractors $\quad 8$

3.1. Ginzburg-Landau equations $\quad 8$

3.2. Kuramoto-Sivashinsky equation $\quad 11$

4. The limit $\varepsilon \rightarrow 0 \quad 14$

4.1. Ginzburg-Landau equations 14

4.2. Kuramoto-Sivashinsky equation 19

5. Reduction to the gradient case $\quad 21$

References $\quad 26$

\section{INTRODUCTION}

The study of systems involving rapid oscillations and their averaging is one of the central subjects of the classical theory of dynamical systems which attracted a great interest during the last century, see, e.g., [2, 14, 28, 38,40,46-48] and references therein. Roughly speaking, it is well-known that the structure of the averaged equations (as well as their validity) is determined by certain resonance interactions. These resonances typically become very complicated when multi-frequency systems are considered, which makes the corresponding averaging problem nontrivial and challenging (e.g., due to the presence of small divisors, see [2] and references therein).

Date: April 12, 2017.

2000 Mathematics Subject Classification. 35B40, 35B45.

Key words and phrases. Dissipative systems, large dispersion, singular perturbation, attractors, averaging. 
Nevertheless, a lot of averaging results are now available for the case of PDEs (which at least formally contain infinitely many frequencies) in both Hamiltonian and dissipative cases, see, e.g., $[8,9,13,15-17,19,22-24,27,28,30,33,34,36,49]$ and references therein.

Very often the analytic structure of the limiting averaged equations is simpler than the structure of the initial problem and this allows us to obtain a reasonable description of the initial dynamics involving rapid oscillations using the averaged equations and singular perturbation technique (see, e.g., $[1,25,51])$. In particular, rapid oscillations may prevent solutions from blowing up in finite time (e.g., for the complex Burgers equation with fast rotation, see $[8,37]$ ), or may give the global in time regularity (e.g., for the 3D Navier-Stokes equations involving strong Coriolis force term, see [9]) or global well-posedness of weak solutions due to the averaging and dispersion effects in large Fourier modes (e.g., for the KdV equations, see $[8,15,16,30]$ ). A similar phenomenon has been observed in [25] concerning three-dimensional high Reynolds number turbulent flows in a periodic box. Specifically, it is shown that a background flow in a periodic domain with large enough spatial average can suppress the turbulent behavior of the flow, viz. it enhances the dissipation (damping) mechanism and effectively reduces the size of the Reynolds number.

However, the opposite effects when rapid oscillations destroy the dissipation mechanism and make the dynamics essentially more complicated are also known (e.g., for the damped hyperbolic equations, see [52]). It is remarkable to observe that, even though the limit averaged equations may remain relatively simple and dissipative, but the global dynamics of the initial system involving rapid oscillations cannot be described by these averaged equations no matter how fast the oscillations are.

This situation somehow emerges in more general class of coupled slow-fast system, where the separation of scales is not strong enough to provide an effective splitting of the state variables into the "slow" and "fast" parts. For instance, this is typical for multi-component systems (where the resonant surfaces may be dense in the phase space) or for systems with chaotic fast component. As a result, an "essential number" of resonant trajectories with the anomalous behavior which is not described by the classically averaged system may appear. This in turn motivates the approaches involving measures and probabilistic arguments. Indeed, as shown in [39] (see also references therein), the limit slow-fast dynamics with uniformly hyperbolic fast part can be effectively described (under some natural assumptions) in terms of a stochastic differential equation. An alternative approach which uses so-called Young measures and treats averaging as a weak limit in the space of measures has been developed in [3-7], see also references therein.

It is worth adding that averaging has turned out to be an appropriate technical tool also for explaining the effective damping mechanism in some energy conservative systems, the so-called Landau damping, see $[18,35,41]$ and [12] for the cases of Vlasov-Poisson system and the 2D Euler equations of incompressible flows respectively.

The aim of the present paper is to study and demonstrate the effect of rapid oscillations induced by large dispersion on the long-time dynamics of dissipative PDEs. For simplicity, we restrict ourselves to consider only the 1D complex Ginzburg-Landau and the KuramotoSivashinsky equations where, on the one hand, the resonances are not complicated and the averaged equations possess a complete description and, on the other hand, a number of nontrivial phenomena which are likely to have general nature can be detected.

To be more precise, we consider the following three model problems on $\mathbb{R}$ :

$$
\begin{array}{r}
\partial_{t} u=(1+i \gamma) \partial_{x}^{2} u+\beta u-(1+i \omega) u|u|^{2}+\varepsilon^{-1} \partial_{x}^{3} u \\
\partial_{t} u=\left(1+i \varepsilon^{-1}\right) \partial_{x}^{2} u+\beta u-(1+i \omega) u|u|^{2} ; \\
\partial_{t} u=-\partial_{x}^{4} u-a \partial_{x}^{2} u+u \partial_{x} u+\varepsilon^{-1} \partial_{x}^{3} u,
\end{array}
$$


subject to periodic boundary conditions with fundamental periodic domain $(-\pi, \pi)$. Here $a, \gamma, \omega$ are given real parameters, $\beta$ is a given complex parameter and $\varepsilon^{-1}$ is a large real parameter. In the first two equations $u$ is assumed to be a complex-valued function: $u=u_{1}+i u_{2}$, and it is real-valued in the third equation.

Based on the analysis given below, we detect three principally different scenarios (paradigms) of how the large dispersion may effect the global dynamics:

Paradigm I (corresponds to equation (1.2)). The dissipation mechanism is not affected by large values of the dispersion parameter $\varepsilon^{-1}$ and the diameter of the global attractor remains bounded, as $\varepsilon^{-1} \rightarrow \infty$. However, the large dispersion limit trivializes the dynamics and the limit averaged equations is a gradient system, up to some simple change of variables. Thus, the global attractor of the limit equation consists of equilibria and heteroclinic connections only (the so-called regular attractor) and no complicated dynamics is possible in the non-averaged equations, when $\varepsilon^{-1}$ is large, see section 5 for more details.

Paradigm II (corresponds to equation (1.1)). Similarly to the previous one, the dissipation mechanism is not affected. However, the complete trivialization of the dynamics, at the infinite dispersion limit, does not happen. Although the global attractor of the limit averaged equations is described by the finite system of ODEs, these ODEs are far from being a gradient system and their dynamics is chaotic (at least for a range of the values of parameters $(\gamma, \beta, \omega)$ that forms an open set in the space of parameters). Then this chaos persists in the initial equations (1.1), when $\varepsilon^{-1}$ is large enough, see Remark 5.7 below, and also [45] for more details.

Paradigm III (corresponds to equation (1.3)). The large dispersion destroys the dissipation mechanism and the diameter of the global attractor grows, as $\varepsilon^{-1} \rightarrow \infty$. In particular, as shown below, see Proposition 4.7, the $L^{2}$-norm of the global attractor associated with equation (1.3) grows proportionally to $\varepsilon^{-1}$, as $\varepsilon^{-1} \rightarrow \infty$. Moreover, we provide here rigorous justification to the numerical investigation, reported in [28]. Note that in this case, we only check that the size of the attractor grows as $\varepsilon \rightarrow 0$. It is an interesting open problem to verify analytically whether or not the other characteristics of the attractor (like dimension, entropies, complexity, etc.) grow as $\varepsilon \rightarrow 0$.

The paper is organized as follows. The resonances and the corresponding infinite dispersion limit equations are presented in section 2 for all three cases of equations (1.1), (1.2) and (1.3). The existence of the corresponding global attractors for these limit equations, as well as the upper bounds for the diameter of these global attractors, are verified in section 3 . The singular limit, $\varepsilon^{-1} \rightarrow \infty$, is studied in section 4 . In particular, the convergence of the global attractors of the Ginzburg-Landau equations to the corresponding global attractors of the infinite dispersion limit equations, as well as the growing lower bounds for the diameter of the global attractor of the Kuramoto-Sivashinsky equation, are established in section 4. Finally, the gradient structure of the infinite dispersion limit equations, corresponding to the Ginzburg-Landau equations (1.2) and its consequences, are presented in section 5 .

\section{Preliminaries}

In this section, we prepare some technical tools which are necessary for our study of equations (1.1)-(1.3). We first note that all of these equations have the form of

$$
\partial_{t} u=A u+\varepsilon^{-1} D u+R(u),
$$

where $A$ is an elliptic operator, $D$ is an antisymmetric operator and $R$ is a non-linearity. Crucial for us is that $A$ commutes with $D$. In addition, in all three cases $e^{\tau D}$ will be $2 \pi$-periodic operator with respect to $\tau$. By these reasons, the natural change of variables $w(t):=e^{-\varepsilon^{-1} t D} u(t)$ formally leads to the equation

$$
\partial_{t} w=A w+F\left(\varepsilon^{-1} t, w\right), \quad F(\tau, w):=e^{-\tau D} R\left(e^{\tau D} w\right)
$$


involving rapid periodic oscillations in time. According to the classical Krylov-Bogolubov averaging principle, we expect that the solution $w=w_{\varepsilon}(t)$ of problem $(2.2)$ will be $\varepsilon$-close (at least on a finite time interval) to the corresponding solution of the averaged equation

$$
\partial_{t} \widehat{w}=A \widehat{w}+N(\widehat{w}), \quad N(w):=\langle F(\tau, w)\rangle=\frac{1}{2 \pi} \int_{0}^{2 \pi} F(\tau, w) d \tau .
$$

In order to justify this scheme, we need to study in more detail the groups $e^{\tau D}$ and the structure of the operators $F$ and $N$ for each of the considered cases. Namely, we introduce the groups of solution operators associated with the auxiliary linear dispersion equations:

$$
\begin{aligned}
& \partial_{t} v=\varepsilon^{-1} \partial_{x}^{3} v, \\
& \partial_{t} v=i \varepsilon^{-1} \partial_{x}^{2} v
\end{aligned}
$$

on $\mathbb{R}$, subject to periodic boundary conditions with fundamental periodic domain $(-\pi, \pi)$.

We denote by $H=L_{p e r}^{2}(-\pi, \pi)$, the space of complex-valued $2 \pi$-periodic square integrable functions, and introduce the family $H^{s}, s \in \mathbb{R}$, of Sobolev spaces of periodic functions with periodic fundamental domain $(-\pi, \pi)$. Let $e_{n}:=e^{i n x}, n \in \mathbb{Z}$, be the standard orthogonal basis in $H$ and let

$$
v=\sum_{n \in \mathbb{Z}} v_{n} e^{i n x}
$$

Neglecting the scalar factor $2 \pi$, we define the $H^{s}$-norm of $v$ as follows:

$$
\|v\|_{H^{s}}^{2}:=\sum_{n \in \mathbb{Z}}\left(n^{2}+1\right)^{s}\left|v_{n}\right|^{2} .
$$

Lemma 2.1. The solution operators $\mathcal{H}_{\varepsilon}(t)$ and $\mathcal{F}_{\varepsilon}(t)$, associated with equations (2.4) and (2.5) respectively, are isometries on the Sobolev spaces $H^{s}$, for any $s \in \mathbb{R}$. Moreover, $e_{n}=e^{\text {inx }}$ are eigenfunctions satisfying

$$
\mathcal{H}_{\varepsilon}(t) e_{n}=e^{-i \varepsilon^{-1} n^{3} t} e_{n}, \quad \mathcal{F}_{\varepsilon}(t) e_{n}=e^{-i \varepsilon^{-1} n^{2} t} e_{n}, \quad n \in \mathbb{Z} .
$$

Finally, these groups of solution operators are $2 \pi \varepsilon$-periodic with respect to time.

Indeed, formulas (2.7) follow immediately from equations (2.4) and (2.5) and all other assertions of the lemma are immediate corollaries of these explicit expressions.

We are going to change the variable $u$ in equations (1.1) and (1.3) using the transformation

$$
u(t)=\mathcal{H}_{\varepsilon}(t) w(t)
$$

and using the transformation

$$
u(t)=\mathcal{F}_{\varepsilon}(t) w(t)
$$

for equation (1.2). Then we will average the obtained rapidly oscillating in time terms. To this end, we need to compute the resonant terms appearing from the nonlinearities. We will do that in the following several subsections.

2.1. Dispersive Ginzburg-Landau equation. We start with the most difficult case of equation (1.1).

Lemma 2.2. Let $w \in H^{s}, s>1 / 2$, and let

$$
F(\tau, w)=F_{G L 1}(\tau, w):=\mathcal{H}_{1}(-\tau) \circ\left(\mathcal{H}_{1}(\tau) w \cdot\left|\mathcal{H}_{1}(\tau) w\right|^{2}\right) .
$$

Then, for every fixed $\tau$, the map $w \rightarrow F(\tau, w)$ is a smooth map from $H^{s}$ to itself, in particular,

$$
\|F(\tau, w)\|_{H^{s}} \leq C_{s}\|w\|_{H^{s}}^{3},
$$


with a constant $C_{s}$ that is independent of $\tau$. Moreover, the operator $F$ is $2 \pi$-periodic with respect to $\tau$, and its time averaging has the form:

$$
N(w)=N_{G L 1}(w)=\langle F(\cdot, w)\rangle:=1 /(2 \pi) \int_{0}^{2 \pi} F(\tau, w) d \tau,
$$

where the operator $N$ has the following explicit form:

$$
N(w)=2 w\|w\|_{H}^{2}+\bar{w}[w, w]-2 w_{0}\left|w_{0}\right|^{2} e_{0}-\sum_{n \neq 0} w_{n}\left(\left|w_{n}\right|^{2}+2\left|w_{-n}\right|^{2}\right) e_{n} .
$$

with $[w, v]:=\sum_{n \in \mathbb{Z}} w_{n} v_{-n}$ and $w=\sum_{n \in \mathbb{Z}} w_{n} e_{n}$. Furthermore,

$$
\begin{aligned}
N(w)=\left(2 w_{0}\left(\|w\|_{H}^{2}-\left|w_{0}\right|^{2}\right)+\right. & \left.\bar{w}_{0}[w, w]\right) e_{0}+ \\
& +\sum_{n \neq 0}\left(w_{n}\left(2\|w\|_{H}^{2}-\left|w_{n}\right|^{2}-2\left|w_{-n}\right|^{2}\right)+\bar{w}_{-n}[w, w]\right) e_{n} .
\end{aligned}
$$

Proof. Indeed, estimate (2.11) follows immediately from the fact that $H^{s}$ is an algebra, for $s>1 / 2$, and that $\mathcal{H}_{1}(\tau)$ are isometries. So, we only need to compute the average of $F(\tau, w)$. Indeed, inserting

$$
u=\mathcal{H}_{1}(\tau) w=\sum_{n \in \mathbb{Z}} e^{-i n^{3} \tau} w_{n} e_{n}
$$

to (2.10) and using that $e_{k} e_{l}=e_{k+l}$, we have

$$
F(\tau, w)=\sum_{n, m, k \in \mathbb{Z}} e^{-i\left(n^{3}+m^{3}+k^{3}-(n+m+k)^{3}\right) \tau} w_{n} \bar{w}_{-m} w_{k} e_{n+m+k} .
$$

Thus, the resonance condition reads

$$
n^{3}+m^{3}+k^{3}=(m+n+k)^{3},
$$

which is equivalent to $(n+m)(m+k)(n+k)=0$. Each of the resonance cases $n+m=0$ and $m+k=0$ give the term

$$
\sum_{n \in \mathbb{Z}} w_{n} e_{n} \sum_{m \in \mathbb{Z}} \bar{w}_{-m} w_{-m}=w \sum_{m \in \mathbb{Z}}\left|w_{m}\right|^{2}=w\|w\|_{H}^{2}
$$

in the right-hand side of $(2.12)$, and the case $n+k=0$ gives the term

$$
\sum_{m \in \mathbb{Z}} \bar{w}_{-m} e_{m} \sum_{n \in \mathbb{Z}} w_{n} w_{-n}=\bar{w}[w, w]
$$

where we have used the fact that $\bar{e}_{m}=e_{-m}$. However, these three families of resonances are not disjoint, but intersect when $m=n=k=0$, for the zero mode (i.e., the corresponding term $w_{0}\left|w_{0}\right|^{2} e_{0}$ is counted three times, so the term $2 w_{0}\left|w_{0}\right|^{2} e_{0}$ should be substracted). Moreover, there are three pairwise intersections at $(m, n, k)=(-l, l, l),(l, l,-l)$ and $(l,-l, l)$, for $l \neq 0$ (all counted twice). These intersections give the remaining terms in formula (2.12). It remains to note that (2.13) is equivalent to (2.12) and Lemma 2.2 is proved.

The next corollary gives the dissipativity of the non-linear operator $N(w)$.

Lemma 2.3. The operator $N=N_{G L 1}$, defined by (2.12), satisfies the following estimate

$$
[N(w), \bar{w}] \geq\|w\|_{H}^{4} .
$$

In particular, the value of $[N(w), \bar{w}]$ is real for every $w \in H^{s}, s>1 / 2$. 
Proof. Indeed, using (2.13), we have

$$
[N(w), \bar{w}]=2\|w\|_{H}^{4}+|[w, w]|^{2}-2\left|w_{0}\right|^{4}-\sum_{n \neq 0}\left|w_{n}\right|^{2}\left(\left|w_{n}\right|^{2}+2\left|w_{-n}\right|^{2}\right),
$$

and the fact that $[N(w), \bar{w}]$ is real is proved. Let us prove (2.16). To this end, we transform the last formula to a more convenient form:

$$
[N(w), \bar{w}]=\|w\|_{H}^{4}+\sum_{n \in \mathbb{Z}}\left|w_{n}\right|^{2} \sum_{m \neq n}\left|w_{m}\right|^{2}+\sum_{n \in \mathbb{Z}} w_{n} w_{-n} \sum_{m \neq \pm n} \overline{w_{m} w_{-m}} .
$$

Using now the Young inequality $|a b| \leq \frac{1}{2}\left(|a|^{2}+|b|^{2}\right)$, we get

$$
\begin{aligned}
& \left|\sum_{n \in \mathbb{Z}} w_{n} w_{-n} \sum_{m \neq \pm n} \overline{w_{m} w_{-m}}\right| \leq \sum_{n \in \mathbb{Z}}\left|w_{n}\right|\left|w_{-n}\right| \sum_{m \neq \pm n}\left|w_{m}\right|\left|w_{-m}\right| \leq \\
& \leq \sum_{n \in \mathbb{Z}}\left|w_{n}\right|\left|w_{-n}\right| \frac{1}{2} \sum_{m \neq \pm n}\left(\left|w_{m}\right|^{2}+\left|w_{-m}\right|^{2}\right)=\sum_{n \in \mathbb{Z}}\left|w_{n}\right|\left|w_{-n}\right| \sum_{m \neq \pm n}\left|w_{m}\right|^{2} \leq \\
& \leq \frac{1}{2} \sum_{n \in \mathbb{Z}}\left(\left|w_{n}\right|^{2}+\left|w_{-n}\right|^{2}\right) \sum_{m \neq \pm n}\left|w_{n}\right|^{2}=\sum_{n \in \mathbb{Z}}\left|w_{n}\right|^{2} \sum_{m \neq \pm n}\left|w_{m}\right|^{2} .
\end{aligned}
$$

This estimate together with (2.17) gives the desired estimate (2.16) and finishes the proof of the lemma.

Remark 2.4. We recall that, according to our notations, $H=L_{p e r}^{2}((-\pi, \pi), \mathbb{C})$,

$$
\|w\|_{H}^{2}=\sum_{n \in \mathbb{Z}}\left|w_{n}\right|^{2}=\frac{1}{2 \pi} \int_{-\pi}^{\pi}|w(x)|^{2} d x
$$

and

$$
(v, w)_{H}=\sum_{n \in \mathbb{Z}} v_{n} \bar{w}_{n}=[v, \bar{w}]=\frac{1}{2 \pi} \int_{-\pi}^{\pi} v(x) \bar{w}(x) d x
$$

(note that $\bar{w}=\overline{\sum_{n \in \mathbb{Z}} w_{n} e_{n}}=\sum_{n \in \mathbb{Z}} \bar{w}_{-n} e_{n}$ ). Thus, inequality (2.16) can be rewritten in the form

$$
\operatorname{Re}(N(w), w)_{H}=(N(w), w)_{H} \geq\|w\|_{H}^{4}
$$

which indeed a standard form of the dissipativity condition.

2.2. Classical Ginzburg-Landau equation. We now formulate the analogue of Lemma 2.2 for equation (1.2).

Lemma 2.5. Let $w \in H^{s}, s>1 / 2$, and let

$$
F(\tau, w)=F_{G L 2}(\tau, w):=\mathcal{F}_{1}(-\tau) \circ\left(\mathcal{F}_{1}(\tau) w \cdot\left|\mathcal{F}_{1}(\tau) w\right|^{2}\right) .
$$

Then, for every fixed $\tau$, the map $w \rightarrow F(\tau, w)$ is a bounded smooth map from $H^{s}$ to itself, and its norm is uniformly bounded with respect to $\tau$ :

$$
\|F(\tau, w)\|_{H_{s}} \leq C_{s}\|w\|_{H^{s}}^{3},
$$

with a constant $C_{s}$ that is independent of $\tau$. Moreover, the operator $F$ is $2 \pi$-periodic with respect to $\tau$, and its time averaging has the form:

$$
N(w)=N_{G L 2}(w):=\left\langle F_{G L 2}(\cdot, w)\right\rangle=2 w\|w\|_{H}^{2}-\sum_{n \in \mathbb{Z}} w_{n}\left|w_{n}\right|^{2} e_{n}=\sum_{n \in \mathbb{Z}} w_{n}\left(2\|w\|_{H}^{2}-\left|w_{n}\right|^{2}\right) e_{n} .
$$


Proof. As before, we only need to compute the average of $F(\tau, w)$. Inserting

$$
u=\mathcal{F}_{1}(\tau) w=\sum_{n \in \mathbb{Z}} e^{-i n^{2} \tau} w_{n} e_{n}
$$

into (2.19), after some elementary calculations we get

$$
F(\tau, w)=\sum_{n, m, k \in \mathbb{Z}} e^{i\left(n^{2}-m^{2}+k^{2}-(n-m+k)^{2}\right) \tau} w_{n} \bar{w}_{m} w_{k} e_{n-m+k} .
$$

Thus, the resonance condition reads

$$
n^{2}-m^{2}+k^{2}=(n-m+k)^{2},
$$

which is equivalent to $(n-m)(k-m)=0$. Thus, we have two families of resonances $n=m$ and $k=m$. Each of them gives the term

$$
\sum_{k \in \mathbb{Z}} w_{k} e_{k} \sum_{n \in \mathbb{Z}} w_{n} \bar{w}_{n}=w\|w\|_{H}^{2}
$$

Observe that these resonance families are not disjoint, and intersect when $(n, k, m)=(l, l, l)$, $l \in \mathbb{Z}$. The common resonance terms are counted twice, so we need to subtract the corresponding term $\sum_{n \in \mathbb{Z}} w_{n}\left|w_{n}\right|^{2} e_{n}$. This gives the desired formula (2.21) and finishes the proof of Lemma 2.5 .

Analogously to the case of equation (1.1), we also have the dissipativity for $N=N_{G L 2}$.

Lemma 2.6. The operator $N=N_{G L 2}$, defined by (2.21), satisfies

$$
[N(w), \bar{w}] \geq\|w\|_{H}^{4}
$$

and again $[N(w), \bar{w}]$ is real.

Proof. Indeed, according to (2.21),

$$
[N(w), \bar{w})]=2\|w\|_{H}^{4}-\sum_{n \in \mathbb{Z}}\left|w_{n}\right|^{4}=\|w\|_{H}^{4}+\sum_{n \in \mathbb{Z}}\left|w_{n}\right|^{2} \sum_{m \neq n}\left|w_{m}\right|^{2} \geq\|w\|_{H}^{4},
$$

and the lemma is proved

2.3. Kuramoto-Sivashinsky equation. We conclude this section by considering the nonlinearity $R(u)=u \partial_{x} u$ associated with the Kuramoto-Sivashinsky equation.

Lemma 2.7. Let $w \in H^{s}, s>1 / 2$, and let

$$
F(\tau, w)=F_{K S}(\tau, w):=\mathcal{H}_{1}(-\tau) \cdot\left(\mathcal{H}_{1}(\tau) w \cdot \partial_{x} \mathcal{H}_{1}(\tau) w\right) .
$$

Then, the operator $F(\tau, \cdot)$ is well-defined and is smooth as an operator from $H^{s}$ to $H^{s-1}$, and the analogue of uniform bounds (2.11) holds, namely

$$
\|F(\tau, w)\|_{H^{s-1}} \leq C_{s}\|w\|_{H^{s}}^{2},
$$

with a constant $C_{s}$ that is independent of $\tau$. Moreover, this operator is $2 \pi$-periodic with respect to time, and its time averaging has the form:

$$
\begin{aligned}
& N(w)=N_{K S}(w):=\left\langle F_{K S}(\cdot, w)\right\rangle= \\
&=\langle w\rangle_{s p} \partial_{x} w+i e_{0} \sum_{n \in \mathbb{Z}} n w_{n} w_{-n}=w_{0} \sum_{n \in \mathbb{Z}} i n w_{n} e_{n}+\left(\sum_{n \in \mathbb{Z}} i n w_{n} w_{-n}\right) e_{0},
\end{aligned}
$$

where $\langle w\rangle_{s p}=w_{0}=1 /(2 \pi) \int_{-\pi}^{\pi} w(x) d x$. 
Proof. As before, we only need to check formula (2.26). Indeed, inserting (2.14) into (2.24), after some elementary calculations, we get

$$
F(\tau, w)=\sum_{n, m \in \mathbb{Z}} i n e^{-i\left(n^{3}+m^{3}-(m+n)^{3}\right) \tau} w_{n} w_{m} e_{n+m}
$$

and, therefore, the resonance condition is

$$
n^{3}+m^{3}=(n+m)^{3},
$$

which gives $n m(n+m)=0$. Moreover, the case $n=0$ gives nothing due to the multiplier $i n$. So, we only have the cases $m=0$ and $m+n=0$ which give the first and the second terms in formula (2.26) respectively. In contrast to the previous cases, these two families intersect only by $(n, m)=(0,0)$, which gives zero effect on $N$ due to the multiplier $i n$. Thus, Lemma 2.7 is proved.

Remark 2.8. In the case of real-valued functions $u$ and $w$ (which is the case for the KuramotoSivashinky equation), we have the additional condition

$$
w_{-n}=\bar{w}_{n}, \quad n \in \mathbb{Z},
$$

and, therefore, the second term in the expression for $N_{K S}(w)$ equals zero identically. Moreover, in the case of the Kuramoto-Sivashinsky equation, we have the additional restriction $w_{0}=0$. Thus, in that case the average of $N_{K S}$ equals zero identically:

$$
N_{K S}(w) \equiv 0 .
$$

\section{Global attractors}

The aim of this section is to formulate and prove some uniform (with respect to $\varepsilon \rightarrow 0$ ) estimates for the global attractors of Ginzburg-Landau equations and verify that their analogue does not take place for the KS equation. The estimates provided here are formal and can be justified in a rigorous way, for instance, by using Galerkin approximation method and then passing to the limit using the appropriate Aubin compactness theorems, see, e.g., $[11,19,50]$ and references therein.

3.1. Ginzburg-Landau equations. We start with the case of equation (1.1).

Theorem 3.1. Equation (1.1) is well-posed in every $H^{s}$, with $s \geq 0$, and the following dissipative estimate holds:

$$
\|u(t)\|_{H^{s}} \leq Q_{s}\left(\left\|u_{0}\right\|_{H^{s}}\right) e^{-\tilde{\gamma} t}+C_{*},
$$

where the monotone function $Q_{s}$ and the positive constants $\tilde{\gamma}$ and $C_{*}$ depend on $s$, but are independent of $\varepsilon$, as $\varepsilon \rightarrow 0$. Moreover, the following smoothing property is valid:

$$
\|u(t)\|_{H^{s}} \leq\left(1+t^{-M_{s}}\right) \tilde{Q}_{s}\left(\left\|u_{0}\right\|_{H}\right), \quad t>0,
$$

where the monotone function $\tilde{Q}_{s}$ and the constant $M_{s}$ are also uniform with respect to $\varepsilon$, as $\varepsilon \rightarrow 0$. Finally, for any two solutions $u_{1}(t)$ and $u_{2}(t)$ of problem (1.1), the following estimate holds:

$$
\left\|u_{1}(t)-u_{2}(t)\right\|_{H} \leq e^{K t}\left\|u_{1}(0)-u_{2}(0)\right\|_{H}
$$

where the constant $K$ depends on the $H$-norms of $u_{i}(0), i=1,2$, but is independent of $\varepsilon$ and $t$.

Proof. Since the assertion of the theorem is more or less standard, we give below only brief derivation of the estimates stated in the theorem, see, e.g., [50] for more details. Moreover, to avoid the technicalities, we derive the dissipative estimate (3.1) for $s=0$. The estimate in a general case, $s \geq 0$, can be obtained in a straightforward way by using the bootstrapping arguments. 
Taking the inner product in $H$ of equation (1.1) with $u$ and integrating by parts, after the straightforward transformations, we have

$$
\frac{1}{2} \frac{d}{d t}\|u(t)\|_{H}^{2}+\left\|\partial_{x} u(t)\right\|_{H}^{2}+\frac{1}{2 \pi}\|u(t)\|_{L^{4}}^{4} \leq \operatorname{Re} \beta\|u(t)\|_{H}^{2} .
$$

Using that $\frac{1}{2 \pi}\|u(t)\|_{L^{4}}^{4} \geq(\operatorname{Re} \beta+1)\|u(t)\|_{H}^{2}-C$, and applying the Gronwall inequality to estimate (3.4), we obtain the following uniform with respect to $\varepsilon$, as $\varepsilon \rightarrow 0$, estimate

$$
\|u(t)\|_{H}^{2}+\int_{t}^{t+1}\left\|\partial_{x} u(t)\right\|_{H}^{2} d t \leq Q\left(\left\|u_{0}\right\|_{H}\right) e^{-\tilde{\gamma} t}+C_{*},
$$

which coincides with the desired estimate (3.1), for $s=0$.

Let us now verify the smoothing property (3.2). For simplicity, we deduce estimate (3.2) for $s=1$ only (for $s>1$, it can be obtained in a standard way using bootstrap arguments). Indeed, due to the embedding theorem

$$
L^{\infty}\left((t, t+1), L^{2}((-\pi, \pi)) \cap L^{2}\left((t, t+1), H^{1}((-\pi, \pi)) \subset L^{6}((t, t+1) \times(-\pi, \pi)),\right.\right.
$$

estimates (3.4) and (3.5) one can establish an estimate for the $L^{2}((t, t+1) \times(-\pi, \pi))$-norm of the nonlinearity. Multiplying now equation (1.1) by $-t \overline{\partial_{x}^{2} u}$ and taking the real part, after the standard transformations, we have

$$
\frac{d}{d t}\left(t\left\|\partial_{x} u(t)\right\|_{H}^{2}\right)+K t\left\|\partial_{x}^{2} u(t)\right\|_{H}^{2} \leq(1+B t)\left\|\partial_{x} u(t)\right\|_{H}^{2}+C t\|u(t)\|_{L^{6}}^{6},
$$

where $K, B$ and $C$ are positive constants. Integrating this relation with respect to time on $[0, t)$, with $t \in(0,1)$, and using (3.5), we deduce estimate (3.2) for $s=1$.

Let us now verify the Lipschitz continuity (3.3). Indeed, let $u_{1}(t)$ and $u_{2}(t)$ be two solutions of equation (1.1) and let $v=u_{1}-u_{2}$. Then, the function $v$ satisfies

$$
\partial_{t} v=(1+i \gamma) \partial_{x}^{2} v+\beta v-(1+i \omega)\left[u_{1}\left|u_{1}\right|^{2}-u_{2}\left|u_{2}\right|^{2}\right]+L \partial_{x}^{3} v, \quad v(0)=u_{1}(0)-u_{2}(0) .
$$

Taking the inner product in $H$ of this equation with $v$ and arguing as before, we get

$$
\frac{1}{2} \frac{d}{d t}\|v\|_{H}^{2}+\left\|\partial_{x} v\right\|_{H}^{2}-\operatorname{Re} \beta\|v\|_{H}^{2} \leq \sqrt{1+w^{2}}\left(\left.\left|u_{1}\right| u_{1}\right|^{2}-u_{2}\left|u_{2}\right|^{2},\left|u_{1}-u_{2}\right|\right) .
$$

The right-hand side of this inequality can be estimated using the Hölder inequality with exponents 3 and $3 / 2$, and the interpolation inequality $\|v\|_{L^{3}} \leq C\|v\|_{L^{2}}^{5 / 6}\|v\|_{H^{1}}^{1 / 6}$ :

$$
\begin{aligned}
& \left(\left.\left|u_{1}\right| u_{1}\right|^{2}-u_{2}\left|u_{2}\right|^{2}|,| u_{1}-u_{2} \mid\right) \leq C\left(\left|u_{1}\right|^{2}+\left|u_{2}\right|^{2},|v|^{2}\right) \leq C\left(\left\|u_{1}\right\|_{L^{6}}^{2}+\left\|u_{2}\right\|_{L^{6}}^{2}\right)\|v\|_{L^{3}}^{2} \leq \\
& \leq C\left(\left\|u_{1}\right\|_{L^{6}}^{2}+\left\|u_{2}\right\|_{L^{6}}^{2}\right)\|v\|_{H}^{5 / 3}\|v\|_{H^{1}}^{1 / 3} \leq C\left(1+\left\|u_{1}\right\|_{L^{6}}^{6}+\left\|u_{2}\right\|_{L^{6}}^{6}\right)\|v\|_{H}^{2}+\left\|\partial_{x} v\right\|_{H}^{2} .
\end{aligned}
$$

Thus, we derived the following estimate:

$$
\frac{1}{2} \frac{d}{d t}\|v\|_{H}^{2} \leq C\left(1+\left\|u_{1}\right\|_{L^{6}}^{6}+\left\|u_{2}\right\|_{L^{6}}^{6}\right)\|v\|_{H}^{2},
$$

where the constant $C$ is independent of $L, u_{1}$ and $u_{2}$. Applying the Gronwall inequality to this relation and using that the space-time $L^{6}$-norm of $u_{i}$ is under control, due to estimate (3.5) and the embedding (3.6), we derive the desired estimate (3.3). The uniqueness of a solution follows from (3.3). Thus, Theorem 3.1 is proved.

Let us consider now the Ginzburg-Landau equation (1.2).

Theorem 3.2. Equation (1.1) is well-posed in every space $H^{s}$, with $s \geq 0$, and estimates (3.1), (3.2) and (3.3) hold uniformly with respect to $\varepsilon$, as $\varepsilon \rightarrow 0$. 
The proof of this theorem follows word by word the proof of the previous one and, thus, is omitted.

Due to Theorems 3.1 and 3.2, the solution semigroups $S_{G L 1}^{\varepsilon}(t)$ and $S_{G L 2}^{\varepsilon}(t)$ associated with equations (1.1) and (1.2) are well-defined in $H$ :

$$
S_{G L i}^{\varepsilon}(t) u(0):=u(t), \quad t \geq 0, \quad u(0) \in H,
$$

where $i=1,2$, and $u(t)$ solves equations (1.1) or (1.2) if $i=1$ or $i=2$, respectively. Moreover, according to estimate (3.1), these semigroups are dissipative in $H^{s}, s \geq 0$ :

$$
\left\|S_{G L i}^{\varepsilon}(t) u_{0}\right\|_{H^{s}} \leq Q_{s}\left(\left\|u_{0}\right\|_{H^{s}}\right) e^{-\tilde{\gamma}_{s} t}+C_{s},
$$

where $Q_{s}, \tilde{\gamma}_{s}$ and $C_{s}$ depend on $s$, but are independent of $L$.

Our next step is to study the global attractors of the introduced semigroups. For the convenience of the reader we start by recalling the definitions related with global attractors, see, e. g., $[11,50]$ for more details.

Definition 3.3. Let $X$ be a Banach space and $S(t): X \mapsto X, t \geq 0$, be a semigroup in $X$. Then, a set $\mathcal{B}$ is an absorbing set for the semigroup $S(t)$ if for any bounded subset $B \subset X$ there exists $T=T(B)$ such that

for all $t \geq T$.

$$
S(t) B \subset \mathcal{B}
$$

A set $\mathcal{B} \subset X$ is an attracting set for the semigroup $S(t)$ if for every bounded $B \subset X$ and every open neighbourhood $\mathcal{O}(\mathcal{B})$ there exists time $T=T(B, \mathcal{O})$ such that

$$
S(t) B \subset \mathcal{O}(\mathcal{B})
$$

for all $t \geq T$. In the case where $\mathcal{B}$ is compact, the attraction property can be rewritten in the equivalent form using the so-called non-symmetric Hausdorff distance. Namely, $\mathcal{B}$ is an attracting set for the semigroup $S(t)$ if, for every bounded $B \subset X$,

$$
\lim _{t \rightarrow \infty} \operatorname{dist}_{X}(S(t) B, \mathcal{B})=0
$$

where the non-symmetric Hausdorff distance between sets $U$ and $V$ of $X$ is defined as follows:

$$
\operatorname{dist}_{X}(U, V):=\sup _{x \in U} \inf _{y \in V}\|x-y\|_{X} .
$$

Finally, a set $\mathcal{A}$ is the global attractor for the semigroup $S(t)$ if the following conditions are satisfied:

1) The set $\mathcal{A}$ is compact in $X$;

2) The set $\mathcal{A}$ is strictly invariant: $S(t) \mathcal{A}=\mathcal{A}$ for all $t \geq 0$;

3) The set $\mathcal{A}$ is an attracting set for the semigroup $S(t)$.

The next corollary gives the existence of global attractors and their uniform bounds with respect to $\varepsilon$, as $\varepsilon \rightarrow 0$, for equations (1.1) and (1.2).

Corollary 3.4. Let $S_{G L 1}^{\varepsilon}(t): H \mapsto H$ and $S_{G L 2}^{\varepsilon}(t): H \mapsto H$ be the solution semigroups generated by equations (1.1) and (1.2), respectively. Then these semigroups possess global attractors $\left(\mathcal{A}_{G L 1}(\varepsilon)\right.$ and $\mathcal{A}_{G L 2}(\varepsilon)$, respectively) in the phase space $H$. Moreover, these global attractors are uniformly bounded with respect to $\varepsilon$, as $\varepsilon \rightarrow 0$, in $H^{s}$, for every $s \geq 0$ :

$$
\left\|\mathcal{A}_{G L 1}(\varepsilon)\right\|_{H^{s}}+\left\|\mathcal{A}_{G L 2}(\varepsilon)\right\|_{H^{s}} \leq C_{s},
$$

where $C_{s}$ depends on $s$, but is independent of $\varepsilon$ (here and below $\|\mathcal{A}\|_{H^{s}}:=\sup _{u \in \mathcal{A}}\|u\|_{H^{s}}$ )

Finally, the global attractors $\mathcal{A}_{G L i}(\varepsilon)$, for $i=1,2$, can be described as follows:

$$
\mathcal{A}_{G L i}(\varepsilon)=\left.\mathcal{K}_{G L i}(\varepsilon)\right|_{t=0},
$$

where $\mathcal{K}_{G L i}(\varepsilon) \subset C_{b}\left(\mathbb{R}, H^{s}\right)$, are the sets of all solutions of equation (1.1) (resp. (1.2)) which are defined for all $t \in \mathbb{R}$ and are bounded. 
Proof. Indeed, according to the abstract theorem on the existence of a global attractor existence, see, e. g., $[11,50]$, we need to verify that

1) Operators $S_{G L i}^{\varepsilon}(t)$ are continuous in $H$, with respect to the initial data, for every fixed $t$;

2) Semigroups $S_{G L i}^{\varepsilon}(t)$ possess compact absorbing sets in $H$.

Note that the first assertion is an immediate corollary of estimate (3.3), so we only need to verify the existence of a compact absorbing set. According to estimate (3.11), the set

$$
\mathcal{B}:=\left\{u \in H,\|u\|_{H}^{2} \leq 2 C_{*}\right\}
$$

is an absorbing set for the semigroups $S_{G L i}^{\varepsilon}(t), i=1,2$. However, this set is not compact in $H$. To overcome this difficulty, we consider the sets

$$
\mathcal{B}_{i}:=S_{G L i}^{\varepsilon}(1) \mathcal{B}, \quad i=1,2 .
$$

Then, due to estimate (3.2), the sets $\mathcal{B}_{i}$ are uniformly (with respect to $\varepsilon$, as $\varepsilon \rightarrow 0$ ) bounded in $H^{s}$, for all $s>0$, and, therefore, they are compact in $H$. Thus, all of the assumptions of the abstract global attractor existence theorem are verified and, consequently, the global attractors $\mathcal{A}_{G L i}(\varepsilon), i=1,2$, exist. Since the global attractor is always a subset of an absorbing set, estimate (3.12) follows from the fact that the absorbing sets $\mathcal{B}_{i}$ are uniformly bounded, with respect to $\varepsilon$, in any $H^{s}$. The description (3.13) also follows from the above mentioned global attractor existence theorem and the corollary is proved.

Remark 3.5. It is well-known (see, e.g., [26]) that the global attractors $\mathcal{A}_{G L 1}$ and $\mathcal{A}_{G L 2}$ are not only smooth (i.e., consist of smooth functions), but also analytic (belong to certain class of Gevrey regularity). Moreover, arguing in a standard way, one can also show that they are uniformly (with respect to $\varepsilon \rightarrow 0$ ) bounded in the appropriate Gevrey norm.

3.2. Kuramoto-Sivashinsky equation. We now turn to the case of Kuramoto-Sivashinsky equation, where the situation is a bit different.

Theorem 3.6. Equation (1.3) possesses a unique solution $u(t) \in H_{0}:=\left\{u \in H,\langle u\rangle_{s p}=0\right\}$ for every $u_{0} \in H_{0}$, and the following estimate holds:

$$
\|u(t)\|_{H}^{2} \leq\|u(0)\|_{H}^{2} e^{-t}+C\left(\varepsilon^{-2}+1\right),
$$

where the positive constant $C$ is independent of $\varepsilon$, but depends on the parameter a.

Proof. We only verify below the dissipative estimate (3.14). The existence and uniqueness can be checked in a usual way (see, e.g., [50]). To this end, we need the following standard lemma (see, e.g., $[21,29,42,44,50]$ ).

Lemma 3.7. For every $M>0$, there exists an odd function $\phi=\phi(M) \in C^{\infty} \cap H_{0}$ such that the following inequality

$$
\left\|\partial_{x}^{2} w\right\|_{H}^{2}-\left(w \partial_{x} \phi, w\right) \geq M\|w\|_{H}^{2}
$$

holds for all $w \in H^{2}$ such that $w(0)=0$.

Following [29], we fix $\phi$ from Lemma 3.7 with $M$ being large enough, consider a family of shifted functions $\phi_{s}(x):=\phi(x-s), s \in \mathbb{R}$, and introduce a functional

$$
F(u(t)):=\min _{s \in[-\pi, \pi]}\left\|u(t)-\phi_{s}\right\|_{H}^{2},
$$

where $u$ is a solution of equation (1.3). Obviously, the minimum exists and is achieved for some value $s^{*}=s^{*}(t)$ which satisfies the following orthogonality condition:

$$
\left(u(t)-\phi_{s^{*}}, \partial_{x} \phi_{s^{*}}\right)=0 .
$$

Let now $v(t):=u(t)-\phi_{s^{*}(t)}$. Then, this function formally satisfies the equation

$$
\partial_{t} u=\partial_{t} v+\partial_{t} s^{*}(t) \partial_{x} \phi_{s^{*}}=-\partial_{x}^{4} v-a \partial_{x}^{2} v+\phi_{s^{*}} \partial_{x} v+\partial_{x} \phi_{s^{*}} v+v \partial_{x} v+\varepsilon^{-1} \partial_{x}^{3} v+f_{\varepsilon}(t)
$$


where $f_{\varepsilon}(t):=-\partial_{x}^{4} \phi_{s^{*}}-a \partial_{x}^{2} \phi_{s^{*}}+\phi_{s^{*}} \partial_{x} \phi_{s^{*}}+\varepsilon^{-1} \partial_{x}^{3} \phi_{s^{*}}$. Multiplying this equation, again formally, by $v$, integrating by $x$ and using the orthogonality condition (3.17), we obtain

$$
\frac{1}{2} \frac{d}{d t}\|v\|_{H}^{2}+\left\|\partial_{x}^{2} v\right\|_{H}^{2}-\frac{1}{2}\left(v \partial_{x} \phi_{s^{*}}, v\right)=a\left\|\partial_{x} v\right\|_{H}^{2}+\left(f_{\varepsilon}, v\right) .
$$

In addition, we claim that the inequality (3.15) with $\phi=\phi_{s^{*}(t)}$ is satisfied for $w=v(t)$, for every $t$ (although $v\left(t, s^{*}\right) \neq 0$ in general). Indeed, from Lemma 3.7, we know that (3.15) with $\phi=\phi_{s^{*}}$ holds for any $w \in H^{2}$ such that $w\left(s^{*}\right)=0$. Let now $K:=v\left(t, s^{*}\right)$ and $w=v-K$. Then, using again the orthogonality condition and the facts that $\langle v\rangle_{s p}=\left\langle\partial_{x} \phi_{s^{*}}\right\rangle_{s p}=0$ and $w\left(s^{*}\right)=0$, we get

$$
\begin{gathered}
\left\|\partial_{x}^{2} v\right\|_{H}^{2}-\left(\partial_{x} \phi_{s^{*}} v, v\right)=\left\|\partial_{x}^{2} w\right\|_{H}^{2}-\left(\partial_{x} \phi_{s^{*}} w, w\right)-2 K\left(\partial_{x} \phi_{s^{*}}, v\right)+K^{2}\left\langle\partial_{x} \phi_{s^{*}}\right\rangle_{s p}= \\
=\left\|\partial_{x}^{2} w\right\|_{H}^{2}-\left(\partial_{x} \phi_{s^{*}} w, w\right) \geq M\|w\|_{H}^{2}= \\
=M\left(\|v\|_{H}^{2}-2 K\langle v\rangle_{s p}+K^{2}\right)=M\|v\|_{H}^{2}+M K^{2} \geq M\|v\|_{H}^{2} .
\end{gathered}
$$

Thus, estimates (3.19) and (3.20) give

$$
\frac{d}{d t}\|v\|_{H}^{2}+\|v\|_{H^{2}}^{2}+M\|v\|_{H}^{2} \leq 2 a\|v\|_{H^{1}}^{2}+2\left|\left(f_{\varepsilon}, v\right)\right| .
$$

It remains to recall that $\left\|f_{\varepsilon}(t)\right\|_{H} \leq C\left(\varepsilon^{-1}+1\right)$ and that, due to interpolation, we have

$$
2 a\|v\|_{H^{1}}^{2} \leq\|v\|_{H^{2}}^{2}+(M-2)\|v\|_{H}^{2}
$$

if $M$ is large enough, depending on the parameter $a$. This gives

$$
\frac{d}{d t}\|v\|_{H}^{2}+\|v\|_{H}^{2} \leq\left\|f_{\varepsilon}\right\|_{H}^{2} \leq C\left(\varepsilon^{-2}+1\right)
$$

and by the Gronwall inequality we formally derive the desired estimate (3.14).

However, there is still an essential gap in the proof, namely, although the minimizer $s^{*}=s^{*}(t)$ for problem (3.16) exists, it is non-necessarily unique. To overcome this problem, we may select one-valued (measurable) branch of the multi-valued function $s^{*}(t)$ (which will be also denoted by $\left.s^{*}(t)\right)$. More essential is the fact that $s^{*}(t)$ may be non-differentiable and even have discontinuities (jumps) at the points where the value $s^{*}(t)$ is not unique. Thus, neither $\partial_{t} s^{*}(t)$ nor $\partial_{t} v(t)$ are properly defined and the multiplication of equation (3.18) by $v(t)$ should be justified. This is done in the following lemma.

Lemma 3.8. Let $u(t) \in H_{0}, t \in[0, T]$, be smooth. Then the function $t \rightarrow F(u(t))$ is absolutely continuous in time and the following formula is valid:

$$
\frac{d}{d t} F(u(t))=\frac{d}{d t}\|v\|_{H}^{2}=2\left(\partial_{t} u(t), v(t)\right), \text { for almost all } t \in[0, T],
$$

where $v(t)=u(t)-\phi_{s^{*}(t)}$ and $s^{*}(t)$ is a minimizer of (3.16).

Proof. We first note that, due to the triangle inequality, the distance function

$$
u \rightarrow \operatorname{dist}\left(u,\left\{\phi_{s}, s \in[-\pi, \pi]\right\}\right)
$$

is Lipschitz continuous with Lipschitz constant one. For this reason, for any $t_{1}, t_{2} \in[0, T]$, we have

$$
\left|\left\|v\left(t_{1}\right)\right\|_{H}-\left\|v\left(t_{2}\right)\right\|_{H}\right| \leq\left\|u\left(t_{1}\right)-u\left(t_{2}\right)\right\|_{H},
$$

so the function $t \rightarrow\|v(t)\|_{H}^{2}$ is Lipschitz continuous despite the fact that $v(t)$ may have jumps. Moreover, if the function $u$ is regular enough, we have

$$
\left\|u\left(t_{1}\right)-u\left(t_{2}\right)\right\|_{H} \leq C\left|t_{1}-t_{2}\right| \text {. }
$$


Inequalities (3.25) and (3.26) show that the function $t \rightarrow\|v(t)\|_{H}^{2}$ is absolutely continuous and, therefore, is differentiable for almost all $t \in[0, T]$. Thus, we only need to find the derivative. To this end, we take $0<t_{2}<t_{1}<T$ such that $\|v(t)\|_{H}^{2}$ is differentiable at $t_{1}$ and $t_{2}$, and we introduce $w=u\left(t_{1}\right)-u\left(t_{2}\right)$. Then using the fact that $F\left(u\left(t_{i}\right)\right)$ is a minimum, we have

$\left\|u\left(t_{1}\right)-\phi_{s_{1}}\right\|_{H}^{2} \leq\left\|u\left(t_{1}\right)-\phi_{s_{2}}\right\|_{H}^{2}=\left\|u\left(t_{2}\right)-\phi_{s_{2}}+w\right\|_{H}^{2}=\left\|u\left(t_{2}\right)-\phi_{s_{2}}\right\|_{H}^{2}+2\left(u\left(t_{2}\right)-\phi_{s_{2}}, w\right)+\|w\|_{H}^{2}$ and, analogously,

$$
\left\|u\left(t_{2}\right)-\phi_{s_{2}}\right\|_{H}^{2} \leq\left\|u\left(t_{1}\right)-\phi_{s_{1}}\right\|_{H}^{2}-2\left(u\left(t_{1}\right)-\phi_{s_{1}}, w\right)+\|w\|_{H}^{2},
$$

where $s_{i}=s^{*}\left(t_{i}\right)$. From these two inequalities we conclude that

$$
\begin{aligned}
2\left(v\left(t_{1}\right), \frac{u\left(t_{1}\right)-u\left(t_{2}\right)}{t_{1}-t_{2}}\right)-\frac{\left\|u\left(t_{1}\right)-u\left(t_{2}\right)\right\|_{H}^{2}}{t_{1}-t_{2}} \leq \frac{\left\|v\left(t_{1}\right)\right\|_{H}^{2}-\left\|v\left(t_{2}\right)\right\|_{H}^{2}}{t_{1}-t_{2}} \leq \\
\quad \leq 2\left(v\left(t_{2}\right), \frac{u\left(t_{1}\right)-u\left(t_{2}\right)}{t_{1}-t_{2}}\right)+\frac{\left\|u\left(t_{1}\right)-u\left(t_{2}\right)\right\|_{H}^{2}}{t_{1}-t_{2}} .
\end{aligned}
$$

Passing now to the limit $t_{1} \rightarrow t_{2}$ in the right-hand side of (3.27), we see that, for almost all $t_{2} \in[0, T]$

$$
\left.\frac{d}{d t}\|v(t)\|_{H}^{2}\right|_{t=t_{2}} \leq 2\left(\partial_{t} u\left(t_{2}\right), v\left(t_{2}\right)\right)
$$

(recall that the existence of the derivative has already been proved). Analogously, passing to the limit $t_{2} \rightarrow t_{1}$ in the left-hand side of inequality (3.27), we get

$$
\left.\frac{d}{d t}\|v(t)\|_{H}^{2}\right|_{t=t_{1}} \geq 2\left(\partial_{t} u\left(t_{1}\right), v\left(t_{1}\right)\right)
$$

for almost all $t_{1} \in[0, T]$. The last two inequalities imply (3.24) and finish the proof of the lemma.

Now, it is not difficult to finish the proof of the theorem. Indeed, the already proved lemma justifies the derivation of the key inequality (3.23) for the case where the solution $u(t)$ is smooth (which will be the case if we start from smooth initial data). The validity of the desired estimate (3.14) for any $u(0) \in H_{0}$ follows then by the usual approximation arguments. Thus, Theorem 3.6 is proved.

Corollary 3.9. Let the assumptions of Theorem 3.6 hold. Then, the Kuramoto-Sivashinsky equation (1.3) possesses a global attractor $\mathcal{A}_{K S}(\varepsilon)$, in the phase space $H_{0}$. Moreover, this global attractor is smooth and

$$
\left\|\mathcal{A}_{K S}(\varepsilon)\right\|_{H^{s}} \leq C_{s}(\varepsilon)
$$

for some positive constant $C$ dependent on $s$, a and $\varepsilon$. In particular, $C_{0}(\varepsilon)=C \varepsilon^{-1}$ with some $C$ independent of $\varepsilon$, but dependent on the parameter $a$.

Proof. Indeed, analogously to (3.2), we have the smoothing property on a finite interval for the parabolic equation (1.3) (see, e.g., [32]):

$$
\|u(t+1)\|_{H^{s}} \leq C\left(1+\|u(t)\|_{H}^{M_{s}}\right)
$$

for some positive $C$ and $M_{s}$. Moreover, the analogue of the Lipschitz estimate (3.3) also holds for this equation (and can be proved analogously to the proof given in Theorem 3.1). Thus, the proof of the corollary repeats word by word the proof of Corollary 3.4 and, for this reason, is omitted.

Remark 3.10. As before, one can show that the global attractor $\mathcal{A}_{K S}(\varepsilon)$ is analytic in the sense of Gevrey class. However, in contrast to the cases of Ginzburg-Landau equations, its Gevrey norm is not uniformly bounded as $\varepsilon \rightarrow 0$. As we will see in the next section, the norms of the global attractors $\mathcal{A}_{K S}(\varepsilon)$ indeed grow, as $\varepsilon \rightarrow 0$. 


\section{The LIMIT $\varepsilon \rightarrow 0$}

The main aim of this section is to study the dependence of the above constructed global attractors on $\varepsilon$, as $\varepsilon \rightarrow 0$. We start with the case of complex Ginzburg-Landau equations (1.2) and (1.1).

4.1. Ginzburg-Landau equations. Following section 2, we change the dependent variable $u$ as follows

$$
\left\{\begin{array}{l}
u(t)=\mathcal{H}_{\varepsilon}(t) w(t) \text { for the case of equation (1.1), } \\
u(t)=\mathcal{F}_{\varepsilon}(t) w(t) \text { for the case of equation (1.2), }
\end{array}\right.
$$

where the isometries $\mathcal{H}_{\varepsilon}$ and $\mathcal{F}_{\varepsilon}$ are defined by (2.7). Then, equations (1.1) and (1.2) read

$$
\partial_{t} w=(1+i \gamma) \partial_{x}^{2} w+\beta w-(1+i \omega) F_{G L 1}(t / \varepsilon, w)
$$

and

$$
\partial_{t} w=\partial_{x}^{2} w+\beta w-(1+i \omega) F_{G L 2}(t / \varepsilon, w),
$$

respectively. The functions $F_{G L 1}$ and $F_{G L 2}$ are defined by (2.10) and (2.19).

Equations (4.2) and (4.3) contain rapidly oscillating in time terms $F_{G L i}(t / \varepsilon, w)$, and we can (formally) write the limit averaged equations:

$$
\partial_{t} \widehat{w}=(1+i \gamma) \partial_{x}^{2} \widehat{w}+\beta \widehat{w}-(1+i \omega) N_{G L 1}(\widehat{w}),
$$

and

$$
\partial_{t} \widehat{w}=\partial_{x}^{2} \widehat{w}+\beta \widehat{w}-(1+i \omega) N_{G L 2}(\widehat{w}),
$$

where the nonlocal operators $N_{G L 1}$ and $N_{G L 2}$ are introduced in Lemma 2.2 and Lemma 2.5. Our first assertion gives the dissipative estimate for the solutions of the limit averaged equations.

Proposition 4.1. Problems (4.4) and (4.5) are globally well-posed for any $\hat{w}(0) \in H^{s}, s \geq 0$, and the corresponding solutions $\hat{w}(t)$ satisfy the analogues of estimates (3.1), (3.2) and (3.3).

Proof. Due to the dissipativity conditions (2.16) and (2.23), in both cases $(N(\widehat{w}), \widehat{w})$ is real and satisfies the dissipative estimate

$$
(N(\widehat{w}), \widehat{w}) \geq\|\widehat{w}\|_{H}^{4} .
$$

Thus, taking a scalar product of the corresponding equation with $\widehat{w}$, we get

$$
\frac{1}{2} \frac{d}{d t}\|\widehat{w}\|_{H}^{2}+\left\|\partial_{x} \widehat{w}\right\|_{H}^{2}+\|\widehat{w}\|_{H}^{4} \leq C\|\widehat{w}\|_{H}^{2}
$$

which gives us the required dissipative estimate in $H$. The proof of the other assertions repeats word by word the proof of Theorem 3.1 and for this reason it is omitted.

Since the averaged problems (4.4) and (4.5) are globally well-posed, the corresponding solution semigroups $\hat{S}_{G L 1}(t): H \mapsto H$ and $\hat{S}_{G L 2}(t): H \mapsto H$ are well defined:

$$
\hat{S}_{G L i}(t) \hat{w}(0):=\hat{w}(t), \quad i=1,2,
$$

where $\hat{w}(t)$ solves problem (4.4) if $i=1$, and problem (4.5) if $i=2$.

Proposition 4.2. The solution semigroups associated with averaged equations (4.4) and (4.5) possess global attractors $\hat{\mathcal{A}}_{G L 1}$ and $\hat{\mathcal{A}}_{G L 2}$, respectively, which are bounded in $H^{s}$, for any $s$. Moreover, these semigroups are invariant with respect to $\mathcal{H}_{1}(s)$ and $\mathcal{F}_{1}(s)$, respectively:

$$
\mathcal{H}_{1}(s) \circ \hat{S}_{G L 1}(t)=\hat{S}_{G L 1}(t) \circ \mathcal{H}_{1}(s), \quad \mathcal{F}_{1}(s) \circ \hat{S}_{G L 2}(t)=\hat{S}_{G L 2}(t) \circ \mathcal{F}_{1}(s),
$$

for all $s \in \mathbb{R}$ and $t \geq 0$, therefore,

$$
\mathcal{H}_{\varepsilon}(s) \widehat{\mathcal{A}}_{G L 1}=\widehat{\mathcal{A}}_{G L 1}, \quad \mathcal{F}_{\varepsilon}(s) \widehat{\mathcal{A}}_{G L 2}=\widehat{\mathcal{A}}_{G L 2},
$$

for all $\varepsilon>0$ and $s \in \mathbb{R}$. 
Proof. Indeed, the existence of global attractors can be verified exactly as in Corollary 3.4, and (4.7) is an immediate corollary of the invariance (4.6). Thus, we only need to check (4.6). In turn, in order to check (4.6), it is enough to verify the invariance of the nonlinearities $N_{G L 1}$ and $N_{G L 2}$ :

$$
\mathcal{H}_{1}(s) N_{G L 1}(w)=N_{G L 1}\left(\mathcal{H}_{1}(s) w\right), \quad \mathcal{F}_{1}(s) N_{G L 2}(w)=N_{G L 2}\left(\mathcal{F}_{1}(s) w\right), \quad s \in \mathbb{R}, \quad w \in H .
$$

Finally, the invariance of $N_{G L i}$ can be easily verified using the explicit formulas (2.7), (2.13) and (2.21). Thus, the proposition is proved.

Our next observation shows that the global attractors $\widehat{\mathcal{A}}_{G L 1}$ and $\widehat{\mathcal{A}}_{G L 2}$ of the limit equations belong to the finite-dimensional invariant hyperplane of the phase space and, thus, can be obtained by solving a system of ODEs.

Proposition 4.3. Let $H_{d}$, where $d \in \mathbb{N}$ be the $(2 D+1)$-dimensional linear subspace

$$
H_{d}:=\left\{\sum_{n=-d}^{d} a_{n} e_{n}, \quad a_{n} \in \mathbb{C}\right\} .
$$

Then the spaces $H_{d}$ are invariant with respect to equations (4.4) and (4.5), for all $d$. Moreover, their global attractors belong to $H_{d}$ provided $d=d(\beta, \omega)$ is large enough:

$$
\widehat{\mathcal{A}}_{G L 1} \subset H_{d}, \widehat{\mathcal{A}}_{G L 2} \subset H_{d} .
$$

In particular, we may take $d=\lfloor\sqrt{\operatorname{Re} \beta}\rfloor+1$ for the case of equation (4.5), here $\lfloor z\rfloor$ denotes the integer part of the number $z$ (the global attractor of (4.5) is trivial if $\operatorname{Re} \beta<0$ ).

Proof. Indeed, the invariance of spaces $H_{d}$ follows immediately from the explicit structure of nonlinearities $N_{G L i}(w)$ given by (2.13) and (2.21). So we only need to prove the embeddings (4.9) if $d$ is large enough. Let us start with the case of the equation (4.4).

Let us consider the equations for $w_{k}$ and $w_{-k}$ from (4.4). Then, multiplying them by $\bar{w}_{k}$ and $\bar{w}_{-k}$ respectively, adding and taking the real part, after the standard calculations, we get

$$
\begin{aligned}
\frac{1}{2} \frac{d}{d t}\left(\left|w_{k}\right|^{2}+\left|w_{-k}\right|^{2}\right)+\left(k^{2}-\operatorname{Re} \beta\right) & \left(\left|w_{k}\right|^{2}+\left|w_{-k}\right|^{2}\right)+ \\
& +\operatorname{Re}\left((1+i \omega)\left([N(w)]_{k} \cdot \bar{w}_{k}+[N(w)]_{-k} \cdot \bar{w}_{-k}\right)\right)=0
\end{aligned}
$$

where we denote by $[N(w)]_{k}$ the $k$ th coordinate of $N(w)$ in the basis $\left\{e_{n}\right\}_{n \in \mathbb{Z}}$. Using the explicit formula (2.13) for the coordinates of $N(w)$, we see that

$$
\left|\operatorname{Re}\left((1+i \omega)\left([N(w)]_{k} \cdot \bar{w}_{k}+[N(w)]_{-k} \cdot \bar{w}_{-k}\right)\right)\right| \leq C \sqrt{1+\omega^{2}}\|w\|_{H}^{2}\left(\left|w_{k}\right|^{2}+\left|w_{-k}\right|^{2}\right),
$$

where the constant $C$ is independent of $k$ and $\omega$. Thus, (4.10) reads

$$
\frac{1}{2} \frac{d}{d t}\left(\left|w_{k}\right|^{2}+\left|w_{-k}\right|^{2}\right)+\left(k^{2}-\operatorname{Re} \beta-C \sqrt{1+\omega^{2}}\|w\|_{H}^{2}\right)\left(\left|w_{k}\right|^{2}+\left|w_{-k}\right|^{2}\right) \leq 0 .
$$

Since the $H$-norm of $w$ is bounded on the global attractor, for sufficiently large $k$, the second term in (4.11) becomes positive and the Gronwall inequality gives

$$
\left|w_{k}(t)\right|^{2}+\left|w_{-k}(t)\right|^{2} \leq e^{-\alpha t}\left(\left|w_{k}(0)\right|^{2}+\left|w_{-k}(0)\right|^{2}\right) \text {, for some } \alpha>0 .
$$

Thus, $w_{k}(t)=w_{-k}(t)=0$ on the global attractor, for $|k|$ large enough, and embedding (4.9) is verified for the case of equation (4.4).

Let us now consider equation (4.5). The situation here is simpler since the expression

$$
[N(w)]_{k} \bar{w}_{k}=\left|w_{k}\right|^{2}\left(2\|w\|_{H}^{2}-\left|w_{k}\right|^{2}\right)
$$

is real and is non-negative and the analogue of (4.11) reads

$$
\frac{1}{2} \frac{d}{d t}\left|w_{k}\right|^{2}+\left(k^{2}-\operatorname{Re} \beta\right)\left|w_{k}\right|^{2} \leq 0 .
$$


Thus, indeed, $w_{k}(t)=0$ on the global attractor if $k^{2}>\operatorname{Re} \beta$, and the proposition is proved.

The next result shows that the distance between the appropriate averaged and non-averaged trajectories is indeed small on the finite time interval.

Theorem 4.4. Let $w_{0} \in H^{s}$, for some $s \geq 1$, and let $w_{\varepsilon}(t)$ and $\widehat{w}(t)$ be the non-averaged and averaged solutions of (4.2) and (4.4), respectively, (or of (4.3) and (4.5), respectively). Assume also that

$$
w_{\varepsilon}(0)=\hat{w}(0) \in H^{s} .
$$

Then, the following estimate holds:

$$
\left\|w_{\varepsilon}(t)-\widehat{w}(t)\right\|_{H^{s}} \leq C_{s} \varepsilon e^{K t},
$$

where the constants $C_{s}$ and $K$ depend on $s$ and on the $H^{s}$ norm of $w(0)$, but are independent of $\varepsilon$, as $\varepsilon \rightarrow 0$.

Proof. Estimate of the form (4.12) is a standard result of the averaging theory and can be referred to as the first Bogolyubov theorem, see, e.g., [14,19,36,48]. However, verifying that the difference between the averaged and non-averaged solutions is of order $O(\varepsilon)$ requires some analysis especially in the case of PDEs. For this reason, we sketch below the proof of this fact for the case of equation (4.2) and $s=1$ (following mainly [27]). The case of equation (4.3) is treated analogously.

We first note that, according to the estimate (3.1) all of the trajectories $w_{\varepsilon}(t)$ and $\widehat{w}(t)$ are uniformly bounded in $H^{1}$. The usual $L^{2}$-parabolic regularity theorem applied to equations (4.2) and (4.4) gives

$$
\left\|\partial_{t} w_{\varepsilon}\right\|_{L^{2}((T, T+1), H)}+\left\|w_{\varepsilon}\right\|_{L^{2}\left((T, T+1), H^{2}\right)} \leq C,
$$

where the constant $C$ depends on the $H^{1}$-norm of the initial data $\hat{w}(0)$, but is independent of $\varepsilon$, as $\varepsilon \rightarrow 0$, and $T \geq 0$, and the same estimate holds for the limit function $\widehat{w}$ as well.

Let us define a function $\theta=\theta_{\varepsilon}(t)$ as the solution of the following equation:

$$
\partial_{t} \theta-(1+i \gamma) \partial_{x}^{2} \theta+\theta=(1+i \omega)\left[F\left(t / \varepsilon, w_{\varepsilon}\right)-N\left(w_{\varepsilon}\right)\right]=: \widetilde{H}\left(t / \varepsilon, w_{\varepsilon}(t)\right), \theta(0)=0 .
$$

We claim that

$$
\left\|\theta_{\varepsilon}(t)\right\|_{H^{1}} \leq C \varepsilon, \quad \text { for all } t \geq 0,
$$

for some constant $C$ that is independent of $\varepsilon$. Indeed, expanding the function $\widetilde{H}\left(\tau, w_{\varepsilon}(t, x)\right)$ into the Fourier series with respect to $\tau$, we have

$$
\widetilde{H}\left(\tau, w_{\varepsilon}(t, x)\right)=\sum_{n \in \mathbb{Z}} e^{i n \tau} F_{n}(t, x),
$$

where

$$
F_{n}(t, x):=\frac{1}{2 \pi} \int_{-\pi}^{\pi} \widetilde{H}\left(\tau, w_{\varepsilon}(t, x)\right) e^{-i n \tau} d \tau
$$

Moreover, by the definition of the operator $N$,

$$
F_{0}=0
$$

and, by the Parseval identity

$$
\sum_{n \in \mathbb{Z}}\left|F_{n}(t, x)\right|^{2}=2 \pi \int_{-\pi}^{\pi}\left|\widetilde{H}\left(\tau, w_{\varepsilon}(t, x)\right)\right|^{2} d \tau, \quad \sum_{n \in \mathbb{Z}}\left|\partial_{x} F_{n}(t, x)\right|^{2}=2 \pi \int_{-\pi}^{\pi}\left|\partial_{x} \widetilde{H}\left(\tau, w_{\varepsilon}(t, x)\right)\right|^{2} d \tau .
$$

Integrating these equalities in $x$, we get

$$
\sum_{n \in \mathbb{Z}}\left\|F_{n}(t)\right\|_{H^{1}}^{2}=2 \pi \int_{-\pi}^{\pi}\left\|\widetilde{H}\left(\tau, w_{\varepsilon}(t)\right)\right\|_{H^{1}}^{2} d \tau
$$


Since $w_{\varepsilon}$ are uniformly bounded in $H^{1}$, we have

$$
\sum_{n \in \mathbb{Z}}\left\|F_{n}(t)\right\|_{H^{1}}^{2} \leq C<\infty
$$

where the constant $C$ is independent of $\varepsilon$ and $t$ (here we have implicitly used (2.20)). Furthermore, using the fact that $\partial_{t} w_{\varepsilon}(t)$ are uniformly bounded in $L^{2}([T, T+1], H)$, and $w_{\varepsilon}(t)$ are uniformly bounded in $L^{2}\left([T, T+1], H^{2}\right)$, with respect to $\varepsilon$, and the explicit formula $(2.10)$ for the nonlinearity $F$, arguing analogously, we can prove that

$$
\sum_{n \in \mathbb{Z}}\left\|\partial_{t} F_{n}(\cdot)\right\|_{L^{2}([T, T+1], H)}^{2} \leq C,
$$

where the constant $C$ is independent of $\varepsilon$ and $T$.

We fix approximate solution $\widehat{\theta}$ of (4.14) in the form

$$
\widehat{\theta}(t):=\sum_{n \neq 0} e^{i n t / \varepsilon}\left(-(1+i \gamma) \partial_{x}^{2}+1+i n / \varepsilon\right)^{-1} F_{n}(t)
$$

(we solve equation (4.14) with respect to the "fast" variable considering the "slow" variable $t$ as a parameter). Then, since the operator $-(1+i \gamma) \partial_{x}^{2}$ generates an analytic semigroup in $H^{s}$, we have

$$
\left\|\left(-(1+i \gamma) \partial_{x}^{2}+1+i n / \varepsilon\right)^{-1}\right\|_{H^{s} \rightarrow H^{s}} \leq C_{s} \frac{\varepsilon}{n},
$$

see, e.g., [32]. Estimate (4.20) together with (4.17) gives

$$
\|\widehat{\theta}(t)\|_{H^{1}} \leq C \varepsilon \sum_{n \neq 0} \frac{\left\|F_{n}(t)\right\|_{H^{1}}}{n} \leq C \varepsilon\left(\sum_{n \neq 0} \frac{1}{n^{2}}\right)^{1 / 2}\left(\sum_{n \neq 0}\left\|F_{n}(t)\right\|_{H^{1}}^{2}\right)^{1 / 2} \leq C^{\prime} \varepsilon .
$$

We now set $\tilde{\theta}:=\theta-\widehat{\theta}$. Then, this function solves

$$
\partial_{t} \tilde{\theta}-(1+i \gamma) \partial_{x}^{2} \tilde{\theta}+\tilde{\theta}=h(t), \quad \tilde{\theta}(0)=-\widehat{\theta}(0),
$$

where

$$
h(t):=-\sum_{n \neq 0} e^{i n t / \varepsilon}\left(-(1+i \gamma) \partial_{x}^{2}+1+i n / \varepsilon\right)^{-1} \partial_{t} F_{n}(t) .
$$

Using now estimate (4.18) together with (4.20), we obtain

$$
\begin{aligned}
\|h\|_{L^{2}([T, T+1], H)} \leq C \varepsilon \sum_{n \neq 0} \frac{\left\|\partial_{t} F_{n}\right\|_{L^{2}([T, T+1], H)}}{n} \leq \\
\leq C \varepsilon\left(\sum_{n \neq 0} \frac{1}{n^{2}}\right)^{1 / 2}\left(\sum_{n \neq 0}\left\|\partial_{t} F_{n}(t)\right\|_{L^{2}([T, T+1], H)}^{2}\right)^{1 / 2} \leq C^{\prime} \varepsilon,
\end{aligned}
$$

where the constant $C^{\prime}$ is independent of $\varepsilon$ and $T$. Estimates (4.21) and (4.23) show that the solution $\tilde{\theta}$ of equation (4.22) satisfies

$$
\|\tilde{\theta}(t)\|_{H^{1}} \leq C \varepsilon,
$$

where the constant $C$ depends on the initial condition $w_{0}$, but is independent of $\varepsilon$ and $t$. Thus, since $\theta=\widehat{\theta}+\tilde{\theta}$, estimate (4.15) is indeed satisfied.

Now, we are ready to finish the proof of the theorem. Let $\tilde{w}:=w_{\varepsilon}-\widehat{w}+\theta$ where $\theta$ solves (4.14). Then, this function satisfies the following equation

$$
\partial_{t} \tilde{w}-(1+i \gamma) \partial_{x}^{2} \tilde{w}=\beta \tilde{w}-(\beta+1) \theta+(1+i \omega)\left[N(\widehat{w})-N\left(w_{\varepsilon}\right)\right], \quad \tilde{w}(0)=0 .
$$


Multiplying this equation by $\partial_{x}^{2} \tilde{w}$ and integrating over $x$, we deduce that

$$
\frac{d}{d t}\|\tilde{w}\|_{H^{1}}^{2} \leq C\left(\|\tilde{w}\|_{H^{1}}^{2}+\|\theta\|_{H^{1}}^{2}+\left\|N\left(w_{\varepsilon}\right)-N(\widehat{w})\right\|_{L^{2}}^{2}\right)
$$

Using that $w_{\varepsilon}$ and $\widehat{w}$ are uniformly bounded in $H^{1}$, one can easily see that

$$
\left\|N\left(w_{\varepsilon}\right)-N(\widehat{w})\right\|_{L^{2}} \leq C\|\tilde{w}\|_{H^{1}}+\|\theta\|_{H^{1}}
$$

and, consequently,

$$
\frac{d}{d t}\|\tilde{w}\|_{H^{1}}^{2} \leq K\|\tilde{w}\|_{H^{1}}^{2}+C\|\theta\|_{H^{1}}^{2}
$$

for some $K$ and $C$ independent of $t$ and $\varepsilon$. Applying the Gronwall inequality for that relation and using (4.15), we deduce estimate (4.12) and finish the proof of the theorem.

As a standard corollary of this theorem (see, for instance, [19] or [31]), we obtain the following result on the convergence of global attractors $\mathcal{A}_{G L 1}(\varepsilon)$ and $\mathcal{A}_{G L 2}(\varepsilon)$ as $\varepsilon \rightarrow 0$.

Corollary 4.5. Let the assumptions of Theorem 4.4 hold, and let $\widehat{\mathcal{A}}_{G L 1}$ and $\widehat{\mathcal{A}}_{G L 2}$ be the global attractors of equations (4.4) and (4.5), respectively. Then the family of global attractors $\mathcal{A}_{G L 1}(\varepsilon)$ (resp. $\mathcal{A}_{G L 2}(\varepsilon)$ ) of equations (1.1) (resp. (1.2)) converge, as $\varepsilon \rightarrow 0$, to the global attractors $\widehat{\mathcal{A}}_{G L 1}$ (resp. $\widehat{\mathcal{A}}_{G L 2}$ ) associated with limit equations (4.4) (resp. (4.5)) in the sense of the upper semicontinuity in $H^{s}, s \geq 0$ :

$$
\lim _{\varepsilon \rightarrow 0} \operatorname{dist}_{H^{s}}\left(\mathcal{A}_{G L 1}(\varepsilon), \widehat{\mathcal{A}}_{G L 1}\right)=\lim _{\varepsilon \rightarrow 0} \operatorname{dist}_{H^{s}}\left(\mathcal{A}_{G L 2}(\varepsilon), \widehat{\mathcal{A}}_{G L 2}\right)=0,
$$

recall that $\operatorname{dist}_{V}(X, Y)$ denotes the non-symmetric Hausdorff distance between sets $X$ and $Y$ in the $V$ norm:

$$
\operatorname{dist}_{V}(X, Y):=\sup _{x \in X} \inf _{y \in Y}\|x-y\|_{V}
$$

Proof. Let $u_{0} \in \mathcal{A}_{G L i}(\varepsilon)$ for $i=1$ or $i=2$. We need to estimate the distance between $u_{0}$ and the global attractor $\hat{\mathcal{A}}_{G L i}$. According to (3.13), there exists a complete bounded trajectory $u_{\varepsilon}(t), t \in$ $\mathbb{R}$, such that $u_{\varepsilon}(t) \in \mathcal{A}_{G L i}(\varepsilon)$, for all $t \in \mathbb{R}$. Moreover, due to (3.12), this trajectory is uniformly bounded by a constant independent of $\varepsilon$ in any space $H^{s}$. Let also $w_{\varepsilon}(t):=\mathcal{H}_{\varepsilon}(-t) u_{\varepsilon}(t)$ (resp. $\left.w_{\varepsilon}(t):=\mathcal{F}_{\varepsilon}(-t) u_{\varepsilon}(t)\right)$. Then, clearly

$$
u_{\varepsilon}(0)=w_{\varepsilon}(0)=u_{0}
$$

and $w_{\varepsilon}(t), t \in \mathbb{R}$, solves equation (4.2) (resp. (4.3)). Take now an arbitrary $T>0$ and consider the solution $\hat{w}(t)$, for $t \geq-T$, of the averaged equation (4.4) (resp. (4.5)) with the initial data

$$
\hat{w}(-T)=w_{\varepsilon}(-T) .
$$

Then, due to estimate (4.12), we have

$$
\left\|u_{0}-\hat{w}(0)\right\|_{H^{s}} \leq\left\|w_{\varepsilon}(0)-\hat{w}(0)\right\|_{H^{s}} \leq C_{s} \varepsilon e^{K T},
$$

where the constants $C$ and $K$ depend only on $s$. On the other hand, since the limit averaged equation possesses a global attractor in $H^{s}$, for any $\delta>0$ we may find $T=T(\delta, s)$ such that

$$
d_{H^{s}}\left(\hat{w}(0), \hat{\mathcal{A}}_{G L i}\right) \leq \delta / 2
$$

for $i=1$ or $i=2$, respectively. Finally, fix $\varepsilon \in\left(0, \varepsilon_{0}\right]$ where $\varepsilon_{0}>0$ is small enough that $C_{s} \varepsilon_{0} e^{K T} \leq \delta / 2$, and using the triangle inequality, we see that

$$
d_{H^{s}}\left(u_{0}, \hat{\mathcal{A}}_{G L i}\right) \leq \delta / 2+\delta / 2=\delta,
$$

and since $\delta>0$ and $u_{0}$ are arbitrary, this inequality proves the desired convergence (4.26), which finishes the proof of the corollary. 
4.2. Kuramoto-Sivashinsky equation. We now turn to the case of Kuramoto-Sivashinsky equation (1.3). In contrast to the case of cubic nonlinearities, the quadratic Kuramoto-Sivashinsky nonlinearity disappears after the averaging and we end up with the non-dissipative averaged equation. This explains why the norm of the global attractor grows with $\varepsilon^{-1}$, as $\varepsilon \rightarrow 0$. To be more precise, the following result holds.

Theorem 4.6. Assume that $a \neq k^{2}, k \in \mathbb{N}$. Then, for every $R>0$ there exists $\varepsilon_{0}=\varepsilon_{0}(R)$ such that $u(t) \equiv 0$ is the only complete trajectory on the global attractor $\mathcal{A}_{K S}(\varepsilon)$, for $\varepsilon \leq \varepsilon_{0}$ which belongs to the $R$-ball in $H$ for all $t \in \mathbb{R}$, i.e, the inequality

$$
\|u(t)\|_{H} \leq R, \quad t \in \mathbb{R}
$$

where $u \in \mathcal{A}_{K S}(\varepsilon)$, implies that $u \equiv 0$.

Proof. Let $u(t)$ be a complete trajectory of the KS equation (1.3) satisfying (4.28). Then, according to the smoothing property, see (3.29), we infer that

$$
\|u(t)\|_{H^{s}} \leq R_{s}, \quad t \in \mathbb{R},
$$

where the constants $C_{s}$ depend on $s$, but are independent of $\varepsilon$.

Let us now introduce a new dependent variable $w(t):=\mathcal{H}_{\varepsilon}(-t) u(t)$. Then, equation (1.3) reads

$$
\partial_{t} w=-\partial_{x}^{4} w-a \partial_{x}^{2} w+F_{K S}(t / \varepsilon, w)
$$

where the operator $F_{K S}$ is defined by (2.24). Using now the fact that $u$ is real, we obtain that $w_{-n}=\bar{w}_{n}$ and, since $\langle u\rangle_{s p}=0$, formula (2.29) gives that the $\tau$-average of $F_{K S}$ equals zero. Thus, the limit averaged equation for (4.30) reads

$$
\partial_{t} \widehat{w}=-\partial_{x}^{4} \widehat{w}-a \partial_{x}^{2} \widehat{w} .
$$

Let us fix $\tau \in \mathbb{R}$ and a solution $\hat{w}(t), t \geq \tau$ of equation (4.31) such that

$$
\hat{w}(\tau)=w(\tau) .
$$

Then, using estimate (4.29) and arguing exactly as in Theorem 4.4, we establish that, for any $\tau \in \mathbb{R}$ and $t \geq 0$, the following estimate holds:

$$
\|w(t+\tau)-\widehat{w}(t+\tau)\|_{H} \leq C \varepsilon e^{K t}, t \geq 0,
$$

where the positive constants $C$ and $K$ are independent of $t, \tau$ and $\varepsilon$.

However, the situation is principally different from the case of Ginzburg-Landau equations, since the averaged equation is now linear and have exponentially growing modes. We claim that inequalities (4.32) and (4.28) imply the estimate

$$
\|u(t)\|_{H} \leq C \varepsilon, \quad t \in \mathbb{R},
$$

for some positive $C$ which may depend on $R$, but is independent of $\varepsilon \rightarrow 0$. Instead of proving estimate (4.33) for the function $u$, we will prove its analogue for $w$ which is equivalent to (4.33) since $\mathcal{H}_{\varepsilon}$ is an isometry in $H$.

Indeed, let $P_{N}$ be an orthogonal projector onto the unstable modes of equation (4.31) (i.e., to the vectors $e_{n}$ with $\left.n^{2}<a\right)$ and $Q_{N}:=1-P_{N}$ is the associated projector to the stable ones (we recall that $\sqrt{a} \notin \mathbb{N}$, so the equilibrium $\widehat{w}=0$ is hyperbolic). Thus, the solution $\widehat{w}$ satisfies the standard exponential dichotomy estimates

$$
\left\{\begin{array}{l}
\left\|P_{N} \widehat{w}(t+\tau)\right\|_{H} \geq C\left\|P_{N} \widehat{w}(\tau)\right\|_{H} e^{\alpha t}, t \geq 0, \\
\left\|Q_{N} \widehat{w}(t+\tau)\right\|_{H} \leq C\left\|Q_{N} \widehat{w}(\tau)\right\|_{H} e^{-\alpha t}, t \geq 0,
\end{array}\right.
$$

for some positive $C$ and $\alpha$. Let us first consider $P_{N}$-component of $w$. Then, using the solution $\widehat{w}$ and estimate (4.34), we get

$$
\left\|P_{N} w(t+\tau)\right\|_{H} \geq\left\|P_{N} \hat{w}(t+\tau)\right\|_{H}-\|w(t+\tau)-\hat{w}(t+\tau)\|_{H} \geq C\left\|P_{N} w(\tau)\right\|_{H} e^{\alpha t}-C \varepsilon e^{K t}
$$


which gives

$$
C\left\|P_{N} w(\tau)\right\|_{H} e^{\alpha t} \leq C \varepsilon e^{K t}+\left\|P_{N} w(t+\tau)\right\|_{H} .
$$

Taking now the supremum over $\tau \in \mathbb{R}$ from the both sides of that inequality, we arrive at

$$
\sup _{\tau \in \mathbb{R}}\left\|P_{N} w(\tau)\right\|_{H} \leq C \varepsilon \frac{e^{K t}}{C e^{\alpha t}-1} .
$$

Fixing here $t$ in an optimal way, we obtain the analogue of (4.33) for the $P_{N}$-component of $w$ :

$$
\sup _{\tau \in \mathbb{R}}\left\|P_{N} w(\tau)\right\|_{H} \leq C^{*} \varepsilon
$$

where $C^{*}$ is independent of $\varepsilon$.

Let us consider now the $Q_{N}$-component of $w$. Then, the analogous arguments lead to the inequality

$$
\left\|Q_{N} w(t+\tau)\right\|_{H} \leq C_{1}\left\|Q_{N} w(\tau)\right\|_{H} e^{-\alpha t}+C_{2} \varepsilon e^{K t}
$$

Taking again the supremum over $\tau \in \mathbb{R}$, we arrive at

$$
\sup _{\tau \in \mathbb{R}}\left\|Q_{N} w(\tau)\right\|_{H} \leq C_{2} \varepsilon \frac{e^{K t}}{1-C_{1} e^{-\alpha t}}, \text { for all } t>\frac{\ln \left(C_{1}+1\right)}{\alpha} .
$$

Minimizing the right-hand side with respect to $t$ over $\left[\ln \left(C_{1}+1\right) / \alpha, \infty\right)$ we obtain

$$
\sup _{\tau \in \mathbb{R}}\left\|Q_{N} w(\tau)\right\|_{H} \leq C^{*} \varepsilon .
$$

Thus, estimate (4.33) is verified.

We see that any trajectory $w(t)$ of equation (4.30) which is contained in the ball of radius $R$ in $H$ should belong to the $C \varepsilon$-neighbourhood of zero equilibrium $w \equiv 0$ (where the constant $C$ depends on $R$, but is independent of $\varepsilon$ ). It remains to note that, since the equilibrium $w \equiv 0$ is hyperbolic and $F_{K S}(\tau, 0)=0,\left.D_{w} F_{K S}(\tau, w)\right|_{w=0}=0$, the usual implicit function theorem shows that the only solution $w(t)$ of (4.30) which belongs to some small $r$-ball of $H$ for any $t(r$ is independent of $\varepsilon !)$ is $w \equiv 0$. Thus, $u \equiv 0$ if $\varepsilon$ is small enough and the theorem is proved.

The proof of Theorem 4.6 indicates particularly that the diameter of the global attractor $\mathcal{A}_{K S}(\varepsilon)$ (say, in the $H$-norm) indeed expands as $\varepsilon \rightarrow 0$ :

$$
\lim _{\varepsilon \rightarrow 0}\left\|\mathcal{A}_{K S}(\varepsilon)\right\|_{H}=\infty
$$

but gives no information about the rate of expansion. The next proposition removes this drawback and shows that the upper bound given by estimate (3.14) is optimal. The proof is based on the well-known fact on the existence of rotating waves for the perturbed KdV equation with periodic boundary conditions, see [43] (see also [28] for the numerical study of the stability of these waves as well as the related attractors).

Proposition 4.7. Let $a>1$ and $\varepsilon$ be small enough. Then, the global attractor $\mathcal{A}_{K S}(\varepsilon)$ satisfies the following estimate:

$$
C_{1} \varepsilon^{-1} \leq\left\|\mathcal{A}_{K S}(\varepsilon)\right\|_{H} \leq C_{2} \varepsilon^{-1}
$$

where the positive constants $C_{1}, C_{2}$ are independent of $\varepsilon$.

Proof. Indeed, the upper bound is an immediate corollary of estimate (3.14), so we only need to establish the lower one. To this end, we do change of variables $u(t)=\varepsilon^{-1} v(t)$ where $v$ is a new dependent variable. Then, equation (1.3) reads

$$
\varepsilon \partial_{t} v=\varepsilon\left(-\partial_{x}^{4} v-a \partial_{x}^{2} v\right)+v \partial_{x} v+\partial_{x}^{3} v
$$

and, finally, introducing the fast variable $\tau=\varepsilon^{-1} t$, we end up with

$$
\partial_{\tau} v=\varepsilon\left(-\partial_{x}^{4} v-a \partial_{x}^{2} v\right)+v \partial_{x} v+\partial_{x}^{3} v
$$


which is a well studied small damped-driven perturbation of the Korteweg de Vries equation. In particular, as shown in [43], if $a>1$ and $\varepsilon$ is small enough, there is a rotating wave solution

$$
v(\tau, x)=V(x-c \tau)
$$

of this equation, where $V=V_{\varepsilon}(\xi)$ is a $2 \pi$-periodic function with zero mean and $c=c_{\varepsilon}$ is a wave speed, both of which have finite non-zero limits as $\varepsilon \rightarrow 0$. Since this rotating wave obviously belongs to the global attractor, it gives the desired lower bound and finishes the proof of the proposition.

\section{ReduCtion to THE GRADIENT CASE}

The main aim of this section is to give more comprehensive study of the dynamics of the complex Ginzburg-Landau equation (1.2) for large dispersion parameter $\varepsilon^{-1}$. As we will see, the large dispersion suppresses the non-trivial dynamics and makes the system gradient-like up to some isometric transformation.

Indeed, the limit averaged equation for (1.2) has the following form, see (4.5):

$$
\frac{d}{d t} w_{n}=-n^{2} w_{n}+\beta w_{n}-(1+i \omega)\left(2 w_{n}\|w\|_{H}^{2}-w_{n}\left|w_{n}\right|^{2}\right), \quad n \in \mathbb{Z},
$$

where $w(t)=\sum_{n \in \mathbb{Z}} w_{n}(t) e_{n}$. Let also $\beta:=\alpha+i \delta, \alpha, \delta \in \mathbb{R}$.

Introducing the new variables $v_{n}$ such that $w_{n}=e^{i A_{n}(t)} v_{n}, n \in \mathbb{Z}$, and the phases $A_{n}(t)$ will be determined later, we get

$$
i v_{n} \frac{d}{d t} A_{n}(t)+\frac{d}{d t} v_{n}=-n^{2} v_{n}+(\alpha+i \delta) v_{n}-(1+i \omega) v_{n}\left(2\|v\|_{H}^{2}+\left|v_{n}\right|^{2}\right), \quad n \in \mathbb{Z} .
$$

Thus, if we fix the phases $A_{n}$ as follows:

$$
A_{n}(t):=\int_{0}^{t}\left(\delta-2 \omega\|v\|_{H}^{2}+\omega\left|v_{n}\right|^{2}\right) d t=\int_{0}^{t}\left(\delta-2 \omega\|w\|_{H}^{2}+\omega\left|w_{n}\right|^{2}\right) d t,
$$

we arrive to the equation with real coefficients

$$
\frac{d}{d t} v_{n}=-n^{2} v_{n}+\alpha v_{n}-2 v_{n}\|v\|_{H}^{2}+v_{n}\left|v_{n}\right|^{2}, \quad n \in \mathbb{Z} .
$$

Moreover, any solution $w(t)$ of (5.1) determines in a unique way the corresponding solution $v(t)$ of (5.4) and vice versa. Therefore, it is sufficient to study equations (5.4) only.

Furthermore, equations (5.4) possess a global Lyapunov function

$$
\mathcal{L}(v):=\|v\|_{H^{1}}^{2}+\|v\|_{H}^{4}-\sum_{n \in \mathbb{Z}} \frac{1}{2}\left|v_{n}\right|^{4}-(\alpha+1)\|v\|_{H}^{2} .
$$

Indeed, as direct calculations show

$$
\frac{d}{d t} \mathcal{L}(v(t))=-2 \sum_{n \in \mathbb{Z}} \frac{d}{d t} v_{n} \frac{d}{d t} \bar{v}_{n}=-2 \sum_{n \in \mathbb{Z}}\left|\frac{d}{d t} v_{n}\right|^{2} .
$$

We are going to apply what is called regular attractors theory (see, e.g. [10]) in order to describe the global attractor $\widehat{\mathcal{A}}_{G L 2}$ of the limit equation (4.5) and the global attractor $\mathcal{A}_{G L 2}(\varepsilon)$ of the perturbed system (4.3), for $\varepsilon^{-1}$ is large enough. However, our situation is slightly more complicated in comparison with the standard theory since equation (5.4) possesses a huge symmetry group

and, for this reason, all of the equilibria are degenerate. Indeed, it follows from the structure of (5.4) that the group $\mathbb{R}^{\infty}$ acting on the phase space by

$$
[\mathcal{S}(\phi) v]_{n}=e^{i \phi_{n}} v_{n}, \quad \phi_{n} \in \mathbb{R}, \quad \phi=\left\{\phi_{n}\right\}_{n \in \mathbb{Z}} \in \mathbb{R}^{\infty}
$$


is a symmetry group of (5.4). For this reason, if $v$ is an equilibrium of equation (5.4), then we automatically have the whole torus of equilibria generated by this symmetry group:

$$
\mathbb{T}_{v}:=\left\{\mathcal{S}(\phi) v, \quad \phi \in \mathbb{R}^{\infty}\right\}
$$

(due to Proposition 4.3, $v_{n} \equiv 0$ for $|n| \geq M=M(\beta)$, for any equilibrium and, consequently, all these tori are, in a fact, finite-dimensional). Thus, the assumption on the hyperbolicity of equilibria should be naturally replaced by the assumption that all of the equilibria tori (5.8) are normally hyperbolic.

In order to verify this normal hyperbolicity and the consequent structure of the global attractor $\widehat{\mathcal{A}}_{G L 2}$ of the limit equation (4.5), we use a simple observation that a real linear subspace

$$
H^{\text {real }}:=\left\{v \in H, v_{n} \in \mathbb{R}, n \in \mathbb{Z}\right\}
$$

is invariant with respect to the limit equation (5.4). Moreover, every initial data $v \in H$ can be reduced to this hyperplane by the appropriate action of the symmetry group $\mathcal{S}(\phi)$. Thus, it is sufficient to check all of the hyperbolicity assumptions for the case of real equations (5.4) and after that obtain the required result for the initial complex phase space by the action of the symmetry group. In particular, one has a natural relation between the real and complex attractors:

$$
\widehat{\mathcal{A}}_{G L 2}=\left\{\mathcal{S}(\phi) \widehat{\mathcal{A}}_{G L 2}^{r e}, \phi \in \mathbb{R}^{\infty}\right\} .
$$

The next lemma gives an explicit description of all possible equilibria for problem (5.4) and establishes their hyperbolicity for generic $\beta$.

Lemma 5.1. The set $\mathcal{R}$ of all equilibria of equation (5.4) consists of $v=0$ and $v \neq 0$ such that the non-zero components $\left\{v_{n}\right\}_{n \in \mathbb{Z}}$ possess the following description: let

$$
N_{0}:=\sum_{n, v_{n} \neq 0} 1, \quad N_{2}:=\sum_{n, v_{n} \neq 0} n^{2} .
$$

Then, for $v_{n} \neq 0$,

$$
\left\{\begin{array}{l}
\|v\|_{H}^{2}=\frac{N_{0}}{2 N_{0}-1} \alpha-\frac{N_{2}}{2 N_{0}-1}>0, \\
\left|v_{n}\right|^{2}=n^{2}+\frac{\alpha-2 N_{2}}{2 N_{0}-1}>0
\end{array}\right.
$$

and every sequence of $v_{n}$ satisfying these conditions gives an equilibrium.

Moreover, a non-zero equilibrium $v$ is not hyperbolic (i.e., the corresponding torus is not normally hyperbolic) if and only if

$$
k^{2}+\frac{\alpha-2 N_{2}}{2 N_{0}-1}=0 \text { for some } k \text { for which } v_{k}=0 .
$$

Finally, zero equilibrium is hyperbolic if and only if $\alpha \neq k^{2}$ for some $k \in \mathbb{Z}$. In particular, all of the equilibria are hyperbolic if $\alpha \neq \mathbb{Z}$.

Proof. Indeed, let $v$ be a non-zero equilibrium. Then, equations (5.4) for $v_{n} \neq 0$ are equivalent to

$$
-n^{2}+\alpha-2\|v\|_{H}^{2}+\left|v_{n}\right|^{2}=0 .
$$

Solving these equations (by using that $\|v\|_{H}^{2}=\sum_{n, v_{n} \neq 0}\left|v_{n}\right|^{2}$ ), we obtain (5.11).

Let us now study the hyperbolicity. As we have already mentioned before, to this end, it is sufficient to consider the case of real equilibrium $v \in \mathcal{R} \cap H^{\text {real }}$ and real perturbation $\theta \in H^{\text {real }}$. For that class of perturbations, the equation of variations reads

$$
\frac{d}{d t} \theta_{n}=-n^{2} \theta_{n}+\alpha \theta_{n}-2 \theta_{n}\|v\|_{H}^{2}-4 v_{n}(v, \theta)+3 \theta_{n} v_{n}^{2}, \quad n \in \mathbb{Z} .
$$


Let us try to find a non-zero eigenvector $\theta \in H^{\text {real }}$ for the right-hand side of these equations which would correspond to the zero eigenvalue.

For $n$th with $v_{n} \neq 0$, equations (5.13) allow to transform the equilibria equation for (5.14) as follows

$$
-4 v_{n}(v, \theta)+2 \theta_{n} v_{n}^{2}=0
$$

or, since $v_{n} \neq 0$, this gives

$$
\theta_{n} v_{n}=2(v, \theta) .
$$

Taking a sum of that equations, we see that $(v, \theta)=2 N_{0}(v, \theta)$ and consequently $(v, \theta)=0$. Equation (5.15) now gives that $\theta_{n}=0$ for all $n$ such that $v_{n} \neq 0$. Let us now consider $k \in \mathbb{Z}$ such that $v_{k}=0$. Then, equation for $\theta_{k}$ reads

$$
\theta_{k}\left(-k^{2}+\alpha-2\|v\|_{H}^{2}\right)=\theta_{k}\left(-k^{2}-\frac{\alpha-2 N_{2}}{2 N_{0}-1}\right)=0
$$

which implies $\theta_{k}=0$ if (5.12) is not satisfied. This implies $\theta \equiv 0$ and non-zero equilibrium $v$ is hyperbolic. Moreover, we see that (5.12) cannot be true if $\alpha$ is not integer, so, for the non-integer $\alpha$ any non-zero equilibrium $v$ is automatically hyperbolic.

Finally, the assertion about zero equilibrium is evident and Lemma 5.1 is proved.

The next lemma gives the stability of the equilibria found in the previous lemma.

Lemma 5.2. Let $\alpha>0$. Then all equilibria $v \in \mathcal{R}$ which have two or more non-zero components $\left(v_{m} \neq 0\right.$ and $v_{k} \neq 0$ for some $\left.m \neq k\right)$ are unstable. The only stable equilibria are the one component ones: $v_{n}=0$ for $n \neq k v_{k} \neq 0$ and $k^{2}<\frac{\alpha}{2}$.

Proof. Let us prove that any equilibrium with two or more non-zero components is unstable. Indeed, let $v \in \mathcal{R}$ be such that $v_{k} \neq 0$ and $v_{m} \neq 0$ for some $k \neq m$. Then, equation (5.13) holds for $n=k$ and $n=m$. Then, (5.14) for these components reads

$$
\left.\frac{d}{d t} \theta_{k}=2 \theta_{k}\left|v_{k}\right|^{2}-4 v_{k}(v, \theta), \quad \frac{d}{d t} \theta_{m}=2 \theta_{m}\left|v_{m}\right|^{2}-4 v_{m}(v, \theta)\right) .
$$

Thus, if we take an inner product of the right-hand side of (5.14) with the non-zero vector $\theta$ such that $\theta_{n}=0$ if $n \neq k, m$ satisfying $(v, \theta)=0$ (exactly for this reason we need at least two non-zero components of $v$ ), the result will be strictly positive: $\theta_{k}^{2} v_{k}^{2}+\theta_{m}^{2} v_{m}^{2}>0$. By the min-max theorem, this means that the corresponding equilibrium is unstable.

Let us now study the equilibria with only one non-zero component $v_{k} \neq 0$ for some $k \neq 0$. Then, (5.13) gives

$$
\left|v_{k}\right|^{2}=\alpha-k^{2}>0
$$

Using this equation in order to simplify (5.14) with $n \neq k$ and using that $v_{n}=0$, we have

$$
\frac{d}{d t} \theta_{n}=\theta_{n}\left(\alpha-2\left|v_{k}\right|^{2}-n^{2}\right)=\theta_{n}\left(2 k^{2}-\alpha-n^{2}\right) .
$$

Finally, the equation for the component with $n=k$ reads

$$
\frac{d}{d t} \theta_{k}=-2 \theta_{k} v_{k}^{2} \text {. }
$$

Thus, the linearization (5.14) at such equilibria is diagonal. Moreover, obviously, all entries on the diagonal will be negative if and only if $2 k^{2}-\alpha<0$, and the lemma is proved.

As a standard corollary of this lemma and the existence of a global Lyapunov function, we obtain the following result. 
Theorem 5.3. Let the parameter $\alpha$ be such that all of the equilibria $v \in \mathcal{R}$ of the equation (5.4) are hyperbolic (see Lemma 5.1). Then, 1) The unstable set of any equilibrium $v_{0} \in \mathcal{R}$

$$
\mathcal{M}_{v_{0}}^{+}:=\left\{v \in H^{1}, \exists v(t), t \in \mathbb{R}, v \text { solves }(5.4), v(0)=v, \quad \lim _{t \rightarrow-\infty} \operatorname{dist}\left(v(t), \mathbb{T}_{v_{0}}\right)=0\right\}
$$

is a finite-dimensional submanifold of the phase space (say, $H^{1}$ ).

2) The global attractor $\widehat{\mathcal{A}}_{G L 2}$ for equations (5.4) is a finite union of the finite-dimensional unstable manifolds:

$$
\widehat{\mathcal{A}}_{G L 2}=\cup_{v_{0} \in \mathcal{R}} \mathcal{M}_{v_{0}}^{+} \text {. }
$$

3) Any trajectory $v(t), t \in \mathbb{R}$, belonging to the global attractor is a heteroclinic orbit between two equilibria $v_{0}^{+}, v_{0}^{-} \in \mathcal{R}$ belonging to different tori:

$$
\lim _{t \rightarrow+\infty}\left\|v(t)-v_{0}^{+}\right\|_{H^{1}}=\lim _{t \rightarrow-\infty}\left\|v(t)-v_{0}^{-}\right\|_{H^{1}}=0, \quad \mathbb{T}_{v_{0}^{+}} \neq \mathbb{T}_{v_{0}^{-}}
$$

4) The global attractor $\widehat{\mathcal{A}}_{G L 2}$ is exponential, i.e., there exists a positive constant $\alpha>0$ and a monotone function $Q$ such that

$$
\operatorname{dist}_{H^{1}}\left(S(t) B, \widehat{\mathcal{A}}_{G L 2}\right) \leq Q\left(\|B\|_{H^{1}}\right) e^{-\alpha t}
$$

for any bounded subset $B$ of the phase space $H^{1}$.

Indeed, the standard regular attractors theory (see, e.g., $[10,20])$ can be applied for the realvalued version of equations (5.4) where all of the equilibria are hyperbolic in a usual sense and the general case can be treated after that by expression (5.10).

Let us return now to the initial equation (5.1). To this end, we just need to put $w_{n}(t)=$ $e^{i A_{n}(t)} v_{n}(t)$, where the phases $A_{n}(t)$ are defined by (5.3). In particular, any equilibrium $v \in \mathcal{R}$ generates a quasi-periodic solution of (5.1). Indeed, in that case

$$
A_{n}(t)=\left(\delta+\omega\left(n^{2}-\alpha\right)\right) t+C_{n}, \quad v_{n} \neq 0,
$$

and we see the quasi-periodic motion with no more than two independent frequencies (generated by $\gamma-\omega \alpha$ and $\omega)$. Thus, invariant tori of equilibria for equation (5.4) correspond to the same tori, but filled by quasi-periodic motions on the level of equation (5.1). Analogously, any heteroclinic orbit connecting the equilibria of equation (5.4) corresponds to the heteroclinic orbits between the aforementioned quasi-periodic motions on the invariant tori. Thus, Theorem 5.3 extends to the initial equation (5.1) just by replacing the tori of equilibria by the tori filled by the aforementioned quasi-periodic motions.

We conclude the section by treating the non-averaged equation (4.3) as a small (of order $\varepsilon$ according to Theorem 4.4) perturbation of the limit equation (5.4). For simplicity, we restrict ourselves to the gradient case $\beta=\alpha \in \mathbb{R}, \omega=0$, see Remark 5.6 below, concerning the general case.

We first note that the non-averaged equations do not possess the symmetry group (5.7) and, consequently the equilibria tori $\mathbb{T}_{w_{0}}$ disappear (in general) for the perturbed equations (4.3).

However, since the (finite-dimensional) invariant tori $\mathbb{T}_{w}, w \in \mathcal{R}$, are normally hyperbolic, they preserve under the small perturbations. To be more precise, in the $\varepsilon$-neighbourhood of every the non-perturbed torus $\mathbb{T}_{w_{0}} \sim \mathbb{T}^{N}$ there exists a smooth $\left(C^{k}\right.$-smooth) invariant torus $\mathbb{T}_{w_{0}}(\varepsilon)$ of the perturbed system (4.3) if $\varepsilon>0$ is small enough. In contrast to the non-perturbed case, the points $w=w(\phi) \in \mathbb{T}_{w_{0}}(\varepsilon), \phi \in \mathbb{T}^{N}$ are no more equilibria, but evolve slowly in time and this evolution is governed by the appropriate system of ODEs

$$
\frac{d}{d t} \phi=\varepsilon f_{\varepsilon}(t, \phi), \quad \phi \in \mathbb{T}^{N}
$$


where the vector field $f_{\varepsilon}$ is of order one as $\varepsilon \rightarrow 0$ :

$$
\left\|f_{\varepsilon}(t, \cdot)\right\|_{C^{k}\left(\mathbb{T}^{N}\right)} \leq C .
$$

We also mention that, although the non-perturbed system (4.3) depends explicitly on time, the invariant tori $\mathbb{T}_{w_{0}}(\varepsilon)$ are independent of time since this equation is invariant under the $\mathcal{F}_{\varepsilon}(s)$-transformations.

Furthermore, according to the general theory, the normally hyperbolic invariant manifolds $\mathbb{T}_{w_{0}}(\varepsilon)$ possess the unstable manifolds $\mathcal{M}_{w_{0}}^{+}(\varepsilon)$ which are $\varepsilon$-close to the unstable manifolds $\mathcal{M}_{w_{0}}^{+}$ of the limit system (moreover, by the above mentioned reasons, they are also independent of $t$ ).

Finally, applying the standard perturbation theory of regular attractors we end up with the following result.

Theorem 5.4. Let the assumptions of Theorem 5.3 hold and let in addition $\beta=\alpha \in \mathbb{R}$ and $\omega=0$. Then, there exists $\varepsilon_{0}>0$ such that, for any $\varepsilon \in\left[0, \varepsilon_{0}\right]$,

1) Every equilibria torus $\mathbb{T}_{w_{0}}, w_{0} \in \mathcal{R}$, generates (in an $\varepsilon$-neighbourhood) a normally hyperbolic invariant torus $\mathbb{T}_{w_{0}}(\varepsilon)$ of the perturbed system (4.3) and the dynamics on it is governed by the slow equations (5.22).

2) The global attractor $\mathcal{A}_{G L 2}(\varepsilon)$ is a finite union of the finite-dimensional unstable manifolds $\mathcal{M}_{w_{0}}^{+}(\varepsilon)$ to that tori:

$$
\mathcal{A}_{G L 2}(\varepsilon)=\cup_{w_{0} \in \mathcal{R}} \mathcal{M}_{w_{0}}^{+}(\varepsilon) .
$$

3) Every trajectory $w(t)$ of the perturbed system (4.3) is a heteroclinic orbit between two trajectories $w^{-}(t) \in \mathbb{T}_{w_{0}^{-}}(\varepsilon)$ and $w^{+}(t) \in \mathbb{T}_{w_{0}^{+}}(\varepsilon)$ belonging to different invariant tori:

$$
\lim _{t \rightarrow+\infty}\left\|w(t)-w^{+}(t)\right\|_{H^{1}}=\lim _{t \rightarrow-\infty}\left\|w(t)-w^{-}(t)\right\|_{H^{1}}=0, \quad w^{ \pm}(t) \in \mathbb{T}_{w_{0}}^{ \pm}(\varepsilon), \mathbb{T}_{w_{0}^{-}} \neq \mathbb{T}_{w_{0}^{+}}
$$

4) The global attractor $\mathcal{A}_{G L 2}(\varepsilon)$ is exponential, i.e., there exist a positive constant $\alpha$ and a monotone function $Q$, such that, for every bounded set $B$ of $H^{1}$,

$$
\operatorname{dist}_{H^{1}}\left(S_{\varepsilon}(t) B, \mathcal{A}_{G L 2}(\varepsilon)\right) \leq Q\left(\|B\|_{H^{1}}\right) e^{-\alpha t},
$$

where $S_{\varepsilon}(t)$ is a solving operator for equation (4.3).

5) The global attractors $\mathcal{A}_{G L 2}(\varepsilon)$ of the equation (4.3) tend to the global attractor $\widehat{\mathcal{A}}_{G L 2}$ of the limit equation (4.5) in the sense of symmetric Hausdorff distance. Moreover, the following estimate holds

$$
\operatorname{dist}_{H^{1}}^{\text {sym }}\left(\mathcal{A}_{G L 2}(\varepsilon), \widehat{\mathcal{A}}_{G L 2}\right) \leq C(\varepsilon)^{\kappa},
$$

where the positive constants $C$ and $\kappa$ are independent of $\varepsilon$.

Indeed, although, in contrast to the general theory, we have now invariant normally hyperbolic tori instead of hyperbolic equilibria, the proof of the result repeats word by word the standard arguments and for this reason it is omitted (see, e.g., [10,20,27] for the details).

Remark 5.5. The last result shows that, for large dispersion parameter $\varepsilon^{-1}$ and $\omega=0$, the dynamics generated by the Ginzburg-Landau equation (1.2) generates three different time scales: 1) rapid oscillations of the phases of the Fourier coefficients (with the frequency proportional to $\varepsilon^{-1}$ ) generated by the group action $\mathcal{F}_{\varepsilon}(t)$;

2 ) order one heteroclinic motion of their amplitudes close to the limit dynamics of (5.4);

3 ) slow drift of their phases (of order $\varepsilon$ ) governed by equations (5.22). We see that the first two types of dynamics are regular. However, the last small drift on the invariant tori, in principle, may be chaotic.

Remark 5.6. The analogue of Theorem 5.4 remains true in the general case $\beta \in \mathbb{C}$ and $\omega \neq 0$. The only difference is that the invariant tori will be filled not by slow motions, but by the motions $\varepsilon$-close to the quasi-periodic ones. 
Remark 5.7. It is natural to ask whether or not the analogous results hold for the equation (1.1). In particular, whether or not the averaged equations (4.4) are in a sense gradient or possess the global Lyapunov function which forbid the complicated dynamics. As shown in [45], the answer on this question is negative and the dynamics of (4.4) is chaotic at least for some values of the parameters $\gamma, \beta$ and $\omega$. Indeed, equations (4.4) obviously possess the 4 -dimensional invariant manifold

$$
\hat{w}=y e_{0}+v\left(e_{1}+e_{-1}\right), \quad y, v \in \mathbb{C}
$$

and the dynamics on this manifold is given by the equations

$$
\left\{\begin{array}{l}
\dot{y}=\beta y-(1+i \omega)\left[y\left(|y|^{2}+4|v|^{2}\right)+2 \bar{y} v^{2}\right] \\
\dot{v}=(\beta-1-i \gamma) v-(1+i \omega)\left[v\left(2|y|^{2}+3|v|^{2}\right)+\bar{v} y^{2}\right] .
\end{array}\right.
$$

Substituting $y=\sqrt{r} e^{i \varphi}, v=\sqrt{\rho} e^{i \psi}, \eta=2(\psi-\varphi)$ and scaling time by the factor of 2 , we arrive at the following 3 -dimensional system:

$$
\left\{\begin{array}{l}
\dot{r}=r[\beta-r-4 \rho-2 \rho(\cos \eta-\omega \sin \eta)], \\
\dot{\rho}=\rho[\beta-1-2 r-3 \rho-r(\cos \eta+\omega \sin \eta)], \\
\dot{\eta}=-\gamma+\omega(\rho-r)+r(\sin \eta-\omega \cos \eta)+2 \rho(\sin \eta+\omega \cos \eta)]
\end{array}\right.
$$

see [45] for the details. As shown there, there are values of parameters $\left(\gamma_{0}, \beta_{0}, \omega_{0}\right)$ for which equations (5.29) possess an equilibrium $\left(r_{0}, \rho_{0}, \eta_{0}\right)$ with 3 zero eigenvalues. Then, the standard bifurcation analysis (also performed in [45]) shows that this system possesses the Shilnikov saddle-focus homoclinic loop at certain values of the parameters and, as a result there is an open region in the space of parameters $(\beta, \gamma, \omega)$ for which the corresponding dynamics generated by (5.29) is chaotic.

Thus, in contrast to (4.5), the averaged equations (4.4) cannot be transformed to a gradient system. Moreover, the standard perturbation arguments show that the chaotic dynamics of the limit averaged equations (4.4) persists at the initial equations (1.1) if $\varepsilon^{-1}$ is large enough.

\section{ACKNOWLEDGEMENTS}

The work of S.Z. was partially supported by the Russian Foundation for Basic Research (projects 14-01-00346 and 15-01-03587) and by the grant 14-41-00044 of RSF. The work of E.S.T. was supported in part by the ONR grant N00014-15-1-2333 and the NSF grants DMS1109640 and DMS-1109645.

\section{REFERENCES}

[1] M. Al-Jaboori and D. Wirosoetisno, Navier-Stokes equations on the $\beta$-plane, Discrete Contin. Dyn. Syst. Ser. B 16 (2011), no. 3, 687-701.

[2] V. Arnold, Mathematical Methods of Classical Mechanics (Graduate Texts in Mathematics, Vol. 60), New York, NY: Springer, 1997.

[3] Z. Artstein, On singularly perturbed ordinary differential equations with measure-valued limits, Math. Bohemica 127 (2002), 139-52.

[4] Z. Artstein, C.W. Gear, I. Kevrikidis, M. Slemrod, and E.S. Titi, Analysis and computation of a discrete $K d V$-Burgers type equation with fast dispersion and slow diffusion, SIAM Journal on Numerical Analysis 49 (2011), no. 5, 2124-2143.

[5] Z. Artstein, I. Kevrikidis, M. Slemrod, and E.S. Titi, Slow observables of singularly perturbed differential equations, Nonlinearity 20 (2007), 2463-2481.

[6] Z. Artstein, J. Linshiz, and E.S. Titi, Young measure approach to computing slowly advansing fast oscillations, Multiscale Model. Simul. 6 (2007), no. 4, 1085-1097.

[7] Z. Artstein and A. Vigodner, ingularly perturbed ordinary differential equations with dynamic limits, Proc. R. Soc. Edinb. A 126 (1996), 541-69.

[8] A. Babin, A. Ilyin, and E.S. Titi, On the regularization mechanism for the periodic Korteweg-de Vries equation, Commun. Pure Appl. Math. 64 (2011), no. 5, 591-648. 
[9] A. Babin, A. Mahalov, and B. Nicolaenko, Global regularity of 3D rotating Navier-Stokes equations for resonant domains, Appl. Math. Lett. 13 (2000), no. 4, 51-57.

[10] A. Babin and M. Vishik, Regular attractors of semigroups and evolution equations, J. Math. Pures Appl. (9) 62 (1983), 441-491.

[11] A. Babin and M. Vishik, Attractors of Evolution Equations, Amsterdam etc.: North-Holland, 1992.

[12] J. Bedrosian and N. Masmoudi, Inviscid damping and the asymptotic stability of planar shear flows in the 2d Euler equations, Publ.math.IHES 122 (2015), 195-300.

[13] A. Bensoussan, J.-L. Lions, and G. Papanicolaou, Asymptotic Analysis for Periodic Structures. Studies in Mathematics and Its Applications. Vol. 5, North-Holland Publ. Amsterdam - New York - Oxford, 1978.

[14] N. Bogolyubov, On Some Statistical Methods in Mathematical Physics, Kiev: Izd. AN USSR, 1945.

[15] J. Bourgain, Fourier transform restriction phenomena for certain lattice subsets and applications to nonlinear evolution equations. II. The KdV-equation, Geom. Funct. Anal. 3 (1993), no. 3, 209-262.

[16] J. Bourgain, Periodic Korteweg de Vries equation with measures as initial data, Selecta Math. (N.S.) 3 (1997), no. 2, 115-159.

[17] J. Bourgain, Global Solutions of Nonlinear Schrödinger Equations. American Mathematical Society Colloquium Publications, Vol. 46, Providence, RI: AMS, 1999.

[18] E. Caglioti and C. Maffei, Time asymptotics for solutions of Vlasov-Poisson equation in a circle, J. Stat. Phys. 92 (1998), no. 1/2.

[19] V. Chepyzhov and M. Vishik, Attractors for Equations of Mathematical Physics, Providence, RI: American Mathematical Society (AMS), 2002.

[20] V. Chepyzhov, M. Vishik, and S. Zelik, Regular attractors and nonautonomous perturbations of them, Sb. Math. 204 (2013), no. 1, 1-42.

[21] P. Collet, J.-P. Eckmann, H. Epstein, and J. Stubbe, A global attracting set for the Kuramoto-Sivashinsky equation, Commun. Math. Phys. 152 (1993), no. 1, 203-214.

[22] J. Colliander, M. Keel, G. Staffilani, H. Takaoka, and T. Tao, Sharp global well-posedness for KdV and modified $K d V$ on $\mathbb{R}$ and $\mathbb{T}$, J. Amer. Math. Soc. 16 (2003), no. 3, 705-749.

[23] J. Colliander, M. Keel, G. Staffilani, H. Takaoka, and T. Tao, Symplectic nonsqueezing of the Korteweg-de Vries flow, Acta Math. 195 (2005), 197-252.

[24] J. Colliander, M. Keel, G. Staffilani, H. Takaoka, and T. Tao, Transfer of energy to high frequencies in the cubic defocusing nonlinear Schrödinger equation, Invent. Math. 181 (2010), no. 1, 39-113.

[25] J. Cyranka, P.B. Mucha, E.S. Titi, and P. Zgliczyński, Stabilizing the long-time behavior of the forced NavierStokes and damped Euler Systems by large mean flow, arXiv:1601.04612[math.AP] (2016).

[26] A. Doelman and E.S. Titi, Regularity of solutions and the convergence of the Galerkin method in the GinzburgLandau equation, Numer. Funct. Anal. Optim. 14 (1993), no. 3-4, 299-321.

[27] M. Efendiev and S. Zelik, The regular attractor for the reaction-diffusion system with a nonlinearity rapidly oscillating in time and its averaging, Adv. Differ. Equ. 8 (2003), no. 6, 673-732.

[28] N. Ercolani, D. McLaughlin, and H. Roitner, Attractors and transients for a perturbed periodic KdV equation: a nonlinear spectral analysis, J. Nonlinear Sci. 3 (1993), 477-539.

[29] J. Goodman, Stability of the Kuramoto-Sivashinsky and related systems, Commun. Pure Appl. Math. 47 (1994), no. 3, 293-306.

[30] Y. Guo, K. Simon, and E.S. Titi, On a nonlinear system of coupled KdV equations, Commun. Math. Sci. 13 (2015), no. 5, 1261-1288.

[31] J. Hale, Asymptotic behavior of gradient dissipative systems, Dynamics of infinite dimensional systems, Proc. NATO Adv. 37 (1987), 123-128.

[32] D. Henry, Geometric Theory of Semilinear Parabolic Equations. Lecture Notes in Mathematics. Vol. 840, Springer-Verlag. Berlin-Heidelberg-New York, 1981.

[33] S. Kozlov, O. Olejnik, and V. Zhikov, Homogenization of Differential Operators and Integral Functionals, Berlin: Springer-Verlag, 1994.

[34] S. Kuksin, Nearly Integrable Infinite-Dimensional Hamiltonian Systems, Berlin: Springer-Verlag, 1993.

[35] L. Landau, On the vibration of the electronic plasma, J. Phys. USSR 10 (1946), no. 25.

[36] B. Levitan and V. Zhikov, Almost Periodic Functions and Differential Equations, Cambridge etc.: Cambridge University Press, 1982.

[37] H. Liu and E. Tadmor, Rotation prevents finite-time breakdown, Phys. D 188 (2004), no. 304, $262-276$.

[38] P. Lochak and C. Meunier, Multiphase Averaging for Classical Systems with Applications to Adiabatic Theorems, Applied Mathematical Sciences 72, Springer-Verlag New York, 1988.

[39] I. Melbourne and A. Stuart, A note on diffusion limits of chaotic skew product flows, Nonlinearity 24 (2011), 1361-1367.

[40] Yu. Mitroploskii, The Averaging Method in Nonlinear Mechanics, Kiev: Naukova Dumka, 1971.

[41] C. Mouhot and C. Villani, On Landau damping, Acta Math. 207 (2011), 29-201. 
[42] B. Nikolaenko, B. Scheurer, and R. Temam, Some global dynamical properties of the Kuramoto-Sivashinsky equations: nonlinear stability and attractors, Phys. D 16 (1985), no. 2, 155-183.

[43] T. Ogawa, Travelling wave solutions to a perturbed Korteweg-de Vries equation, Hiroshima Math. J. 24 (1994), no. 2, 401-422.

[44] F. Otto, Optimal bounds on the Kuramoto-Sivashinsky equation., J. Funct. Anal. 257 (2009), no. 7, 21882245.

[45] I. Ovsyannikov, D. Turaev, and S. Zelik, Bifurcation to chaos in the complex Ginzburg-Landau equation with large 3rd order dispersion, Modeling and Analysis of Information Systems 22 (2015), no. 3, 327-366.

[46] G. Pavliotis and A. Stuart, Multiscale Methods. Averaging and Homogenization, Texts in Applied Mathematics, 53. Springer, New York, 2008.

[47] H. Poincaré, Les Méthodes Nouvelles de la Mécanique Céleste. Tome I. Solutions Périodiques. Non-existence des Intégrales Uniformes. Solutions Asymptotiques, Paris: Gauthier-Villars et Fils, 1892.

[48] J. Sanders, F. Verhulst, and J. Murdock, Averaging Methods in Nonlinear Dynamical Systems, 2nd ed., New York, NY: Springer, 2007.

[49] E. Tadmor and T. Tao, Velocity averaging, kinetic formulations, and regularizing effects in quasi-linear PDEs, Comm. Pure Appl. Math. 60 (2007), no. 10, 1488-1521.

[50] R. Temam, Infinite-dimensional Dynamical Systems in Mechanics and Physics, 2nd ed., New York, NY: Springer, 1997.

[51] D. Wirosoetisno, Navier-Stokes equations on a rapidly rotating sphere, Discrete Contin. Dyn. Syst. Ser. B 20 (2015), no. 4, 1251-1259.

[52] S. Zelik, Global averaging and parametric resonances in damped semilinear wave equations, Proc. R. Soc. Edinb., Sect. A, Math. 136 (2006), no. 5, 1053-1097.

1 University of Surrey, Department of Mathematics, Guildford, GU2 7XH, United Kingdom, A.KOSTIANKO@SURREY.AC.UK, S.ZELIK@SURREY.AC.UK.

${ }^{2}$ Department of Mathematics, Texas A \& M University, 3368 TAMU, College Station, TX 778433368, USA, TITi@MATH.TAMU.EDU.

3 Weizmann Institute of Science, Department of Computer Science and Applied Mathematics, REHOVOT, 76100, ISRAEL, EDRISS.TITI@WEIZMANN.AC.IL. 